\title{
AVALIAÇÃO DA BIOMASSA FOLIAR DE MORANGUEIRO HIDROPÔNICO EM DIFERENTES AMBIENTES PROTEGIDOS
}

\author{
Leaf biomass evaluation of hydroponic strawberry in greenhouses
}

Edilson Costa1, Paulo Ademar Martins Leal ${ }^{2}$

\begin{abstract}
RESUMO
Realizaram-se experimentos na Faculdade de Engenharia Agrícola na Universidade Estadual de Campinas, utilizando quatro cultivares de morangueiro [Fragaria x ananassa (Weston) Duchesne ex Rozier], quatro sistemas de produção hidropônica (canal de $100 \mathrm{~mm}$, canal de $150 \mathrm{~mm}$, canal de $150 \mathrm{~mm}$, com vaso contendo fibra de coco e tubo vertical contendo casca de arroz carbonizada) e três ambientes protegidos (casa-de-vegetação sem resfriamento evaporativo do ar e sem injeção aérea de $\mathrm{CO}_{2}$, casa-de-vegetação com injeção aérea de $\mathrm{CO}_{2}$ e sem resfriamento evaporativo do ar e casa-de-vegetação com injeção aérea de $\mathrm{CO}_{2}$ e resfriamento evaporativo do ar). Foram avaliados os parâmetros foliares massa fresca foliar (MFF) e massa seca foliar (MSF) em gramas e área foliar (AF) em $\mathrm{mm}^{2}$, correlacionando à biomassa da planta. $\mathrm{O}$ melhor sistema foi o de canais de $150 \mathrm{~mm}$ com vaso contendo fibra de coco. O melhor ambiente foi o de resfriamento evaporativo do ar e injeção aérea de $\mathrm{CO}_{2}$.
\end{abstract}

Termos para indexação: Fragaria x ananassa, hidroponia, estufas, climatização.

\begin{abstract}
Analyses were made in the experimental field of the Agricultural Engineering College at State University of Campinas (Unicamp). Four varieties of strawberry [Fragaria $x$ ananassa (Weston) Duchesne ex Rozier] were tested in four hydroponics production systems $(100 \mathrm{~mm}$ and $150 \mathrm{~mm}$ channels and $150 \mathrm{~mm}$ channel with a vase containing coconut fiber and a vertical pipe containing carbonized rice rusk). Three greenhouses were tested: i) a greenhouse without air evaporative cooling nor $\mathrm{CO}_{2}$ aerial injection, ii) a greenhouse with $\mathrm{CO}_{2}$ aerial injection and without air evaporative cooling and iii) a greenhouse with $\mathrm{CO}_{2}$ aerial injection and air evaporative cooling. It was analyzed the leaf fresh (MFF) and dry mass in gram (MSF) and the leaf area in $\mathrm{mm}^{2}(\mathrm{AF})$. The best hydroponics system was $150 \mathrm{~mm}$ channel with a vase containing coconut fiber. The best environment condition was the one with air evaporative cooling and aerial injection of $\mathrm{CO}_{2}$.
\end{abstract}

Index terms: Fragaria x ananassa, hydroponics, greenhouses, climatization

(Recebido em 15 de outubro de 2007 e aprovado em 13 de maio de 2008)

\section{INTRODUÇÃO}

Conforme explicitado por Costa (2004) há poucos estudos sobre o comportamento do morangueiro em sistema hidropônico no Brasil, considerando-se a grande extensão que possui o país e as necessidades de conhecimento sobre a adaptação dessa cultura nesse sistema de produção.

Os parâmetros de crescimento, área e massa foliar caracterizam a biomassa da planta, de maneira que podem ser usados para determinar mudanças na assimilação de carboidratos pela planta, durante uma estação do ano (BUTLER et al., 2002), onde a área foliar mede o potencial da planta acumular biomassa aérea e a massa seca foliar determina a capacidade da planta em aumentar seu peso seco através da fotossíntese.
Segundo Pires et al. (1999) a avaliação da produção de plantas de morangueiro requer as análises quantitativas do seu crescimento, destacando que o índice que relaciona a área foliar com o desenvolvimento vegetal é um parâmetro fisiológico muito utilizado na análise de crescimento das plantas, uma vez que a taxa fotossintética depende diretamente dele.

As modificações microclimáticas ambientais das casas-de-vegetação, ou seja, das coberturas plásticas para produção vegetal, tem promovido reflexos positivos às culturas, pois aumenta a produtividade de frutos, a área foliar e a qualidade dos produtos produzidos (BURIOL et al., 1997; SEGOVIA et al., 1997).

Dentro de ambientes protegidos o morangueiro pode ser desenvolvido em sistemas convencionais (solo) ou em sistema hidropônicos utilizando ou não substratos.

\footnotetext{
'Engenheiro Agrícola, Doutor, Professor - Unidade Universitária de Aquidauana/UUA - Universidade Estadual de Mato Grosso do Sul/UEMS - Rodovia Aquidauana, CEPA, Km 12 - Zona Rural - Cx. P. 25 - 79200-000 - Aquidauana, MS - mestrine@uems.br

2Engenheiro Agrícola, Doutor, Professor - Conselho Integrado de Tecnologia de Processos/CITP - Faculdade de Engenharia Agrícola/FEAGRI Universidade Estadual de Campinas/UNICAMP - Barão Geraldo - Cx. P. 6011 - 13083-970 - Campinas, SP - pamleal@agr.unicamp.br
} 
No solo da casa-de-vegetação, os canteiros são formados com três ou quatro fileiras de produções com espaçamento entre plantas de $25 \mathrm{~cm}$ e espaçamentos entre fileiras de $30 \mathrm{~cm}$. Em sistemas hidropônicos pode-se conseguir um maior adensamento das plantas quando se utilizam sistemas verticais com substratos ou sistemas de bancadas com filme de solução nutritiva (FERNANDES JÚNIOR, 2001).

Gusmão (2000), na região de Jaboticabal/SP, avaliando duas variedades de morangueiro, Campinas e Seascape, em sistema hidropônico NFT (Nutrient Film Technique), com canais de $100 \mathrm{~mm}$, em ambiente protegido, observou que a variedade Campinas apresentou produção, crescimento vegetativo e emissão de estolhos maior que a variedade Seascape.

Segundo Nunes (2000), citado por Carrijo et al. (2004) a utilização de fibra de coco como substrato, em produções sem solo, promove um excelente desenvolvimento à planta, principalmente por reter uma maior quantidade de umidade e propiciar um melhor desenvolvimento do sistema radicular.

Objetivou-se, neste trabalho, avaliar a biomassa foliar de quatro cultivares de morangueiro [Fragaria $x$ ananassa (Weston) Duchesne ex Rozier] (Campinas, Seascape, Sweet Charlie e Tudla), dentro de diferentes ambientes protegidos, utilizando vários sistemas hidropônicos.

\section{MATERIAL E MÉTODOS}

Experimentos com morangueiro, das cultivares Campinas, Seascape, Sweet Charlie e Tudla, foram desenvolvidos em três diferentes ambientes protegidos e quatro sistemas hidropônicos, durante o período de abril de 2002 a março de 2003, na região de Campinas/SP. Os ambientes foram três casas-de-vegetação: sem resfriamento evaporativo do ar e sem injeção de $\mathrm{CO}_{2}$ (Ambiente 1 - A1); com apenas injeção de $\mathrm{CO}_{2}$ e sem resfriamento evaporativo do ar (Ambiente 2 - A2) e resfriamento evaporativo do ar e injeção de $\mathrm{CO}_{2}$ (Ambiente 3 - A3). Utilizaram-se os sistemas hidropônicos NFT em bancadas tipo "A" com canais de 100mm (Sistema 1-S1), com canais de $150 \mathrm{mmm}$ (Sistema $2-\mathrm{S} 2$ ) e com canais de $150 \mathrm{~mm}$ com vaso contendo fibra de coco (Sistema $3-\mathrm{S} 3$ ) e tubos verticais contendo casca de arroz carbonizada (Sistema 4-S4).

As casas-de-vegetação de estrutura metálica de aço com dimensões de $6,50 \mathrm{~m}$ de largura, 11,0m de comprimento, $3,0 \mathrm{~m}$ de pé-direito, $2,0 \mathrm{~m}$ de altura acima do pé-direito e altura da cumeeira de $5,0 \mathrm{~m}$, possuíam uma porta com largura de $1,17 \mathrm{~m}$ e altura de $2,05 \mathrm{~m}$, tendo um perímetro de $36,0 \mathrm{~m}$, com área de piso de $71,5 \mathrm{~m}^{2}$ e volume de $286 \mathrm{~m}^{3}$. O telhado do tipo duas águas, com inclinação de $31,6^{\circ}$, constituído por plástico transparente difusor de luz (PEBD - Polietileno de Baixa Densidade) com 150 microns de espessura, tratado contra raios ultravioleta. Todos os ambientes possuíam cortinas termorefletoras.

As cortinas internas termorefletoras $60 \%$ de sombra, possuíam abertura e fechamento realizados por motores reversos, através de controlador com leitura de um foto sensor LDR (Light Dependent Resistor) ou RVL (Resistor Variável de Luz).

O sistema de resfriamento evaporativo instalado no Ambiente A3 foi composto pelo meio poroso de celulose e dois exaustores que exauriam o ar interno.

Foram coletadas as temperaturas no período de dezembro de 2002 a março de 2003, nos horários das 9:00h e 15:00h, no ambiente externo e nas casas-de-vegetação. A média geral às 9:00h, no ambiente externo e nos ambientes $\mathrm{A} 1, \mathrm{~A} 2$ e $\mathrm{A} 3$ foram, respectivamente, $25,8^{\circ} \mathrm{C}, 26,8^{\circ} \mathrm{C}, 26,5^{\circ} \mathrm{C}$ e $25,2^{\circ} \mathrm{C}$. A média geral às $15: 00 \mathrm{~h}$, no ambiente externo e nos ambientes A1, A2 e A3 foram, respectivamente, $29,8^{\circ} \mathrm{C}$, $30,5^{\circ} \mathrm{C}, 30,2^{\circ} \mathrm{C}$ e $27,4^{\circ} \mathrm{C}$.

As mudas foram adquiridas em bandejas de poliestireno da empresa Multiplanta (Andradas/MG), possuindo certificado de isenção de patógenos, com aproximadamente 5,0 (cinco) centímetros de altura, no mês de dezembro de 2001, transferidas para os sistemas de cultivo em abril de 2002 e cultivadas até março de 2003. Aproximadamente, a cada dois meses foram realizadas limpezas nas plantas, retirando-se folhas mortas e restos de cultura.

O tratamento fitossanitário constituiu-se de aplicação de defensivos para controle de antracnose, ácaros, pulgões e lagarta-rosca.

Nos sistemas S1 e S2, as mudas foram transplantadas diretamente nos canais de cultivo, enquanto no sistema S3 as mudas foram acomodadas em vasos e esses alocados dentro do canal de cultivo. No sistema S4, as mudas foram transplantadas diretamente à casca de arroz carbonizada. $\mathrm{O}$ sistema $\mathrm{S} 1$ possuía 12 canais de $100 \mathrm{~mm}$ com 15 plantas cada. Os sistemas S2 e S3 possuíam 6 canais de 150mm com 15 plantas cada, onde o sistema S3 continha vasos com substrato para suporte das plantas (fibra de coco). O sistema S4 continha 04 linhas com sete plantas cada, totalizando 28 plantas por tubo, os quais foram preenchidos com casca de arroz carbonizada, sistema utilizado por Fernandes Júnior (2001).

Utilizou-se a solução nutritiva recomendada por Fernandes Júnior (2001), sendo que a mesma foi refrigerada por um sistema de expansão. 
Nos sistemas S1, S2 e S3 o fornecimento da solução nutritiva foi controlado por um temporizador, responsável pela automação do tempo de circulação da solução. Das $6 \mathrm{~h} 00$ às $9 \mathrm{~h} 00$, o sistema funcionava 10 minutos e permanecia parado $30 \mathrm{~min}$. Das $9 \mathrm{~h} 00$ às $11 \mathrm{~h} 00$, o sistema funcionava 10 minutos e permanecia parado 20 min. Das 11 h00 às 16h00, o sistema funcionava 10 minutos e permanecia parado 10 min. Das $16 \mathrm{~h} 00$ às $18 \mathrm{~h} 00$, o sistema funcionava 10 minutos e permanecia parado $20 \mathrm{~min}$. Durante a noite o sistema funcionava 10 minutos em cada hora. Para o sistema S4, a solução nutritiva foi fornecida uma ou duas vezes ao dia, onde cada coluna de cultivo possuía um registro de regulagem de vazão.

$\mathrm{O}$ dióxido de carbono $\left(\mathrm{CO}_{2}\right)$, injetado diretamente nas folhas das plantas, foi aplicado em três períodos: das $9 \mathrm{~h} 30$ às $10 \mathrm{~h} 00$, das $10 \mathrm{~h} 15$ às $10 \mathrm{~h} 45 \mathrm{e}$ das $11 \mathrm{~h} 00$ às $11 \mathrm{~h} 30$. No ambiente A2, quando a temperatura permitia, o $\mathrm{CO}_{2}$ era aplicado com as cortinas laterais fechadas. A concentração de $\mathrm{CO}_{2}$ foi mantida por volta de 1200ppm.

Foram coletados dados de massa fresca foliar (MFF) e massa seca foliar (MSF) em gramas e área foliar do trifolíolo (AF), em milímetros quadrados, de 10 folhas de morangueiro de diferentes plantas, escolhidas aleatoriamente, evitando-se folhas muitas jovens e folhas em senescência. As coletas foram realizadas em três períodos, assim definidos maio, junho e julho (MJJ, período 1) no início de produção de frutos; agosto, setembro e outubro (ASO, período 2) no pico de produção de frutos e novembro, dezembro, janeiro, fevereiro e março (NDJFM, período 3) no término de produção de frutos.

Após a coleta, determinava-se a massa fresca e a área foliar de cada folha, sendo posteriormente levada à estufa para secagem. A área foliar foi determinada pelo método do peso do material em função de uma área conhecida (BENINCASA, 1988) e as massas através de balança analítica.

O delineamento experimental utilizado foi em parcelas subdivididas, no esquema 3 x 4 (três ambientes $\mathrm{x}$ quatro sistemas hidropônicos), onde as parcelas principais foram os ambientes de cultivo e as subparcelas foram os sistemas hidropônicos, para cada cultivar de morangueiro. Adotou-se como repetição a folha, sendo no total 10 repetições.

Os dados foram submetidos à análise de variância e as médias ao teste de Tukey, ao nível de 5\% de probabilidade. Quando havia interação significativa entre ambientes e sistemas fêz-se o desdobramento, estudando-se um fator dentro do outro. As análises foram realizadas pelo programa computacional ESTAT
(Sistema de Análise Estatística - UNESP/FCAVJ, 1994) da UNESP (1994).

\section{RESULTADOS E DISCUSSÃO}

Analisando a cultivar Campinas (Tabelas 1 e 2), observa-se através da Tabela 1, para AF, MFF e MSF, nos três períodos estudados, que o ambiente climatizado (ambiente A3) proporcionou maiores valores que os demais ambientes, resultados que corroboram as explicitações de Buriol et al. (1997) e Segovia et al. (1997) em relação à modificações ambientais. Os dados, também revelaram que em ambientes não-climatizados, comparando-se os ambientes A1 e A2, a aplicação de $\mathrm{CO}_{2}$ (ambiente $\mathrm{A} 2$ ) não proporcionou efeitos desejáveis, ou seja, não houve aumento de área foliar, possivelmente não acarretando aumento da taxa fotossintética (PIRES et al., 1999).

Nos ambientes A1 e A2, o processo convectivo do ar foi menor que no ambiente climatizado (COSTA et al., 2004; CUNHA \& ESCOBEDO, 2003), sendo mais marcante o efeito estufa, promovendo maior evapotranspiração em função de um maior gasto de energia e, conseqüentemente, acarretando menor desenvolvimento das plantas.

Nos diferentes períodos, os sistemas hidropônicos apresentaram variações em relação à AF. No entanto, observa-se uma predominância do sistema $\mathrm{S} 3$ em relação aos demais, apresentando uma maior AF, resultado que concorda com Nunes (2000), citado por Carrijo et al. (2004), a respeito dos resultados benéficos da fibra de coco, para melhor desenvolvimento da planta. Tanto para a MFF como para a MSF destacou o sistema S3 no período de MJJ, onde se observa que, no último período de coleta NDJFM, os sistemas praticamente não diferiram (Tabela 1).

Fernandes Júnior (2001) coloca que, no sistema vertical (S4), as produções de frutos e de estolhos por planta são menores que nos sistemas de bancadas, fato também observado, nesse experimento, para os parâmetros foliares no pico de produção de "frutos".

Em síntese, verifica-se para a cultivar Campinas, através dos parâmetros estudados, que o ambiente A3 e o sistema S3 podem ter promovido um maior deslocamento e alocação de carboidratos, na parte aérea da planta. Butler et al. (2002) destacam que esse processo, na planta, promove maior acúmulo de biomassa, capacitando a mesma a aumentar sua taxa fotossintética.

No último período NDJFM, para os desdobramentos da AF, observa-se que, dentro dos ambientes A1 e A2, os sistemas praticamente apresentaram os mesmos resultados, no entanto, dentro do ambiente A3, destacou-se o sistema S4. Para os desdobramentos da MFF e da MSF, os sistemas praticamente não diferenciaram nos ambientes (Tabela 2). 
Tabela 1 - Área foliar (AF), massa fresca foliar (MFF) e massa seca foliar (MSF) para a cultivar Campinas nos ambientes (A) e nos Sistemas (S), nos períodos de maio a julho (MJJ), agosto a outubro (ASO), novembro a março (NDJFM) ${ }^{1}$.

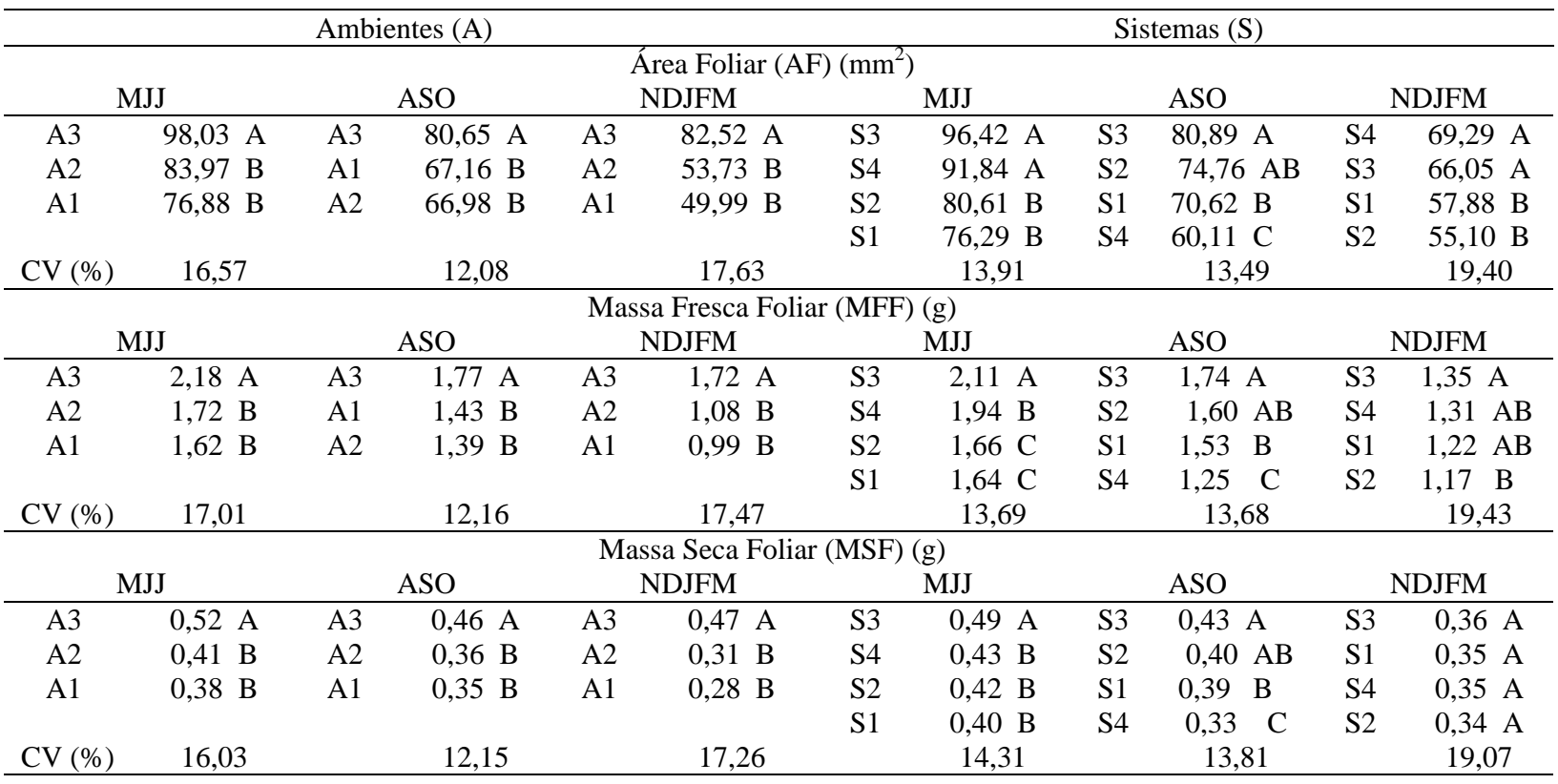

${ }^{1}$ Médias seguidas de pelo menos uma letra comum, nas colunas de cada variável, não diferem significativamente entre si, pelo teste de Tukey $(\mathrm{P}<0,05)$.

Tabela 2 - Desdobramentos dos sistemas (S) dentro dos ambientes (A) e desdobramentos dos ambientes (A) dentro dos sistemas (S), para a área foliar (AF) em $\mathrm{mm}^{2}$, para a massa fresca foliar (MFF) e massa seca foliar (MSF) em gramas, da cultivar Campinas ${ }^{1}$.

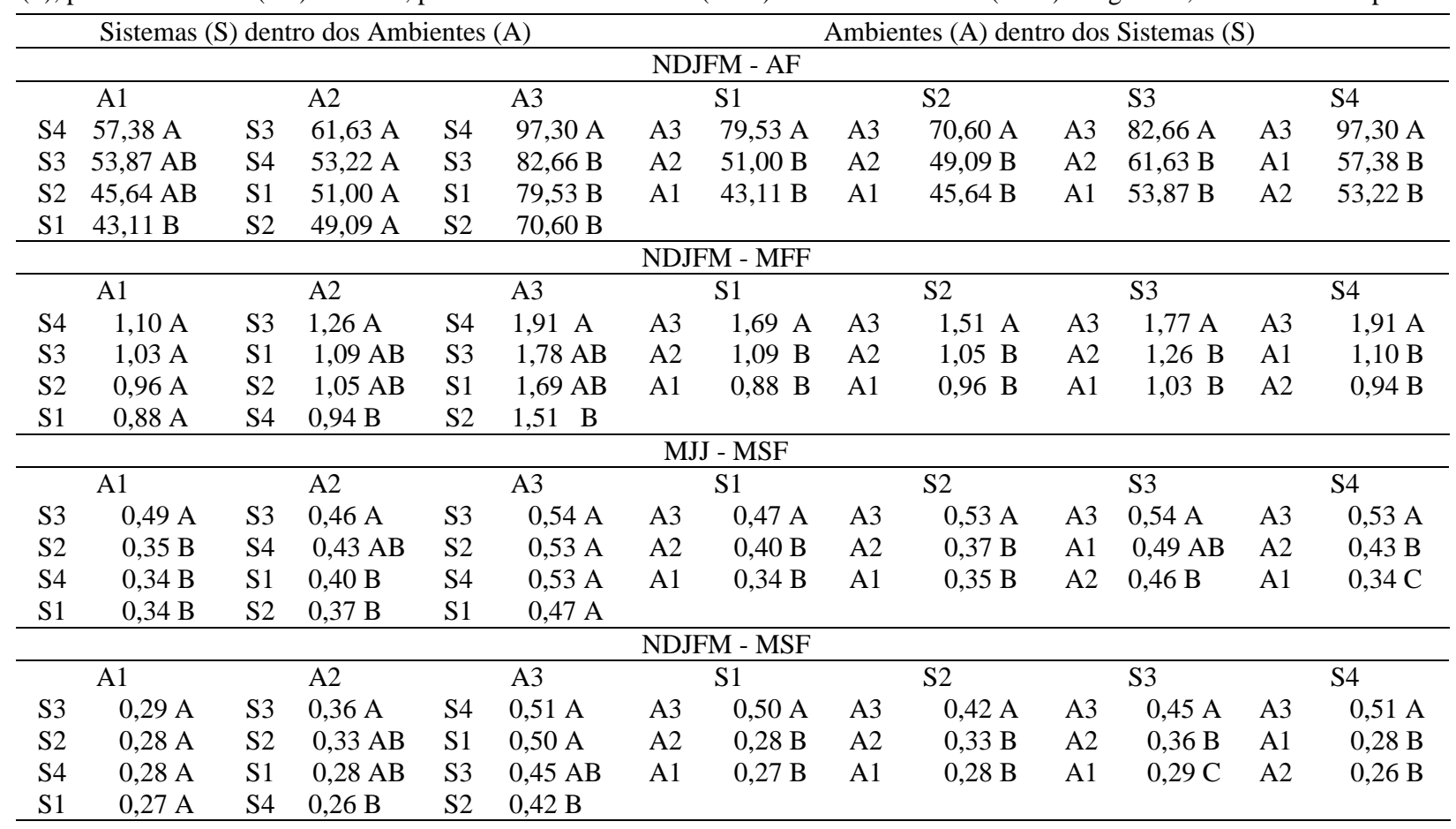

${ }^{1}$ Médias na mesma coluna, seguidas de mesma letra, não diferem entre si, pelo teste de Tukey $(\mathrm{P}<0,05)$. 
Nessa mesma Tabela verifica-se que, tanto para a AF como para a MFF, para todos os sistemas o ambiente A3 destacou-se (BURIOL et al., 1997; SEGOVIA et al., 1997). No período de MJJ dentro do ambiente A1, o sistema S3 apresentou maior MSF que os demais, e, no ambiente A2, o sistema S3 não diferiu do sistema S4.

Para a área foliar (AF) e massa fresca foliar (MFF), nos períodos de MJJ e ASO, e para a massa seca foliar (MSF) no período de ASO os desdobramentos dos sistemas (S) dentro dos ambientes (A) e desdobramentos dos ambientes (A) dentro dos sistemas (S), da cultivar Campinas, não foram significativos.

A partir dos parâmetros foliares estudados para a cultivar Campinas, verifica-se, que tanto para os efeitos principais (ambientes) como para os efeitos secundários (sistemas), com o passar dos períodos e o desenvolvimento da cultura seus valores foram diminuindo, diferenciando de valores encontrados por Butler et al. (2002), o qual explicita que não houve diferenças ao longo do período de coleta, que foi de outubro a maio, período de baixas temperaturas no Hemisfério Norte. Essa diminuição nos valores encontrados nos experimentos realizados em Campinas-SP deve-se ao fato do mesmo estar inserido numa região que apresenta grandes aumentos de temperatura ao longo do período estudado, em que, no período de dezembro de 2002 a março de 2003, a média foi de $25,8^{\circ} \mathrm{C}$ às $9 \mathrm{~h} 00$, solicitando da planta um maior gasto de energia, promovendo uma maior evapotranspiração e, conseqüentemente, menor acúmulo de carboidratos.

Analisando a cultivar Seascape (Tabelas 3, 4, 5 e 6), observa-se através da Tabela 3 que, para os três parâmetros avaliados AF, MFF, MSF, os resultados mostraram-se similares aos resultados apresentados para a cultivar Campinas, ou seja, destacou-se o ambiente A3, em que a modificação no ambiente de produção promoveu melhor desenvolvimento da planta concordando com explanações de Buriol et al. (1997) e Segovia et al. (1997). Destacou-se, também, o sistema S3, que possuía fibra de coco, dessa forma concordando com comentários de Nunes (2000), citado por Carrijo et al. (2004) a respeito da utilização desse substrato para as plantas.

Tabela 3 - Área foliar (AF), massa fresca foliar (MFF) e massa seca foliar (MSF) para a cultivar Seascape nos ambientes (A) e nos Sistemas (S), nos períodos de maio a julho (MJJ), agosto a outubro (ASO), novembro a março (NDJFM) $)^{1}$.

\begin{tabular}{|c|c|c|c|c|c|c|c|c|c|c|c|}
\hline \multicolumn{6}{|c|}{ Ambientes (A) } & \multicolumn{6}{|c|}{ Sistemas (S) } \\
\hline \multicolumn{12}{|c|}{ Área Foliar (AF) $\left(\mathrm{mm}^{2}\right)$} \\
\hline \multicolumn{2}{|c|}{ MJJ } & \multicolumn{2}{|c|}{ ASO } & \multicolumn{2}{|c|}{ NDJFM } & \multicolumn{2}{|c|}{ MJJ } & \multicolumn{2}{|c|}{ ASO } & \multicolumn{2}{|c|}{ NDJFM } \\
\hline A3 & $88,34 \mathrm{~A}$ & A3 & $62,74 \mathrm{~A}$ & A3 & $52,42 \mathrm{~A}$ & S4 & $100,16 \mathrm{~A}$ & S3 & $71,83 \mathrm{~A}$ & S3 & $56,87 \mathrm{~A}$ \\
\hline $\mathrm{A} 2$ & $73,15 \mathrm{~B}$ & A2 & $54,82 \mathrm{AB}$ & A2 & $40,89 \mathrm{~B}$ & S3 & $94,89 \mathrm{~A}$ & $\mathrm{~S} 2$ & $58,67 \mathrm{~B}$ & S4 & $48,60 \mathrm{~B}$ \\
\hline \multirow[t]{2}{*}{ A1 } & $67,48 \mathrm{~B}$ & A1 & $53,30 \mathrm{~B}$ & A1 & $38,27 \mathrm{~B}$ & $\mathrm{~S} 2$ & $56,16 \mathrm{~B}$ & S4 & $49,33 \mathrm{C}$ & $\mathrm{S} 2$ & $35,16 \mathrm{C}$ \\
\hline & & & & & & $\mathrm{S} 1$ & $54,15 \mathrm{~B}$ & $\mathrm{~S} 1$ & $47,98 \mathrm{C}$ & S1 & $34,79 \mathrm{C}$ \\
\hline $\mathrm{CV}(\%)$ & 28,19 & & 25,16 & & 22,98 & & 21,46 & & 18,52 & & 22,68 \\
\hline \multicolumn{12}{|c|}{ Massa Fresca Foliar (MFF) (g) } \\
\hline \multicolumn{2}{|c|}{ MJJ } & \multicolumn{2}{|c|}{ ASO } & \multicolumn{2}{|c|}{ NDJFM } & \multicolumn{2}{|c|}{ MJJ } & \multicolumn{2}{|c|}{ ASO } & \multicolumn{2}{|c|}{ NDJFM } \\
\hline A3 & $2,14 \mathrm{~A}$ & A3 & $1,65 \mathrm{~A}$ & A3 & $1,18 \mathrm{~A}$ & S3 & $2,44 \mathrm{~A}$ & S3 & $1,78 \mathrm{~A}$ & S3 & $1,33 \mathrm{~A}$ \\
\hline A2 & $1,71 \mathrm{~B}$ & A1 & $1,28 \mathrm{~B}$ & A1 & $0,79 \mathrm{~B}$ & $\mathrm{~S} 4$ & $2,40 \mathrm{~A}$ & $\mathrm{~S} 2$ & $1,49 \mathrm{~B}$ & S4 & $1,03 \mathrm{~B}$ \\
\hline \multirow[t]{2}{*}{ A1 } & $1,62 \mathrm{~B}$ & A2 & $1,20 \mathrm{~B}$ & A2 & $0,79 \mathrm{~B}$ & $\mathrm{~S} 2$ & $1,27 \mathrm{~B}$ & $\mathrm{~S} 4$ & $1,12 \mathrm{C}$ & $\mathrm{S} 2$ & $0,73 \mathrm{C}$ \\
\hline & & & & & & $\mathrm{S} 1$ & $1,19 \mathrm{~B}$ & $\mathrm{~S} 1$ & $1,11 \mathrm{C}$ & S1 & $0,60 \mathrm{C}$ \\
\hline $\mathrm{CV}(\%)$ & 28,31 & & 25,47 & & 24,52 & & 21,71 & & 19,01 & & 24,18 \\
\hline \multicolumn{12}{|c|}{ Massa Seca Foliar (MSF) (g) } \\
\hline \multicolumn{2}{|c|}{ MJJ } & \multicolumn{2}{|c|}{ ASO } & \multicolumn{2}{|c|}{ NDJFM } & \multicolumn{2}{|c|}{ MJJ } & \multicolumn{2}{|c|}{ ASO } & \multicolumn{2}{|c|}{ NDJFM } \\
\hline A3 & $0,51 \mathrm{~A}$ & A3 & $0,43 \mathrm{~A}$ & A3 & $0,33 \mathrm{~A}$ & S3 & $0,59 \mathrm{~A}$ & S3 & $0,44 \mathrm{~A}$ & S3 & $0,39 \mathrm{~A}$ \\
\hline $\mathrm{A} 2$ & $0,43 \mathrm{~B}$ & A1 & $0,32 \mathrm{~B}$ & $\mathrm{~A} 2$ & $0,22 \mathrm{~B}$ & $\mathrm{~S} 4$ & $0,57 \mathrm{~A}$ & $\mathrm{~S} 2$ & $0,38 \mathrm{~B}$ & S4 & $0,28 \mathrm{~B}$ \\
\hline \multirow[t]{2}{*}{ A1 } & $0,41 \mathrm{~B}$ & A2 & $0,30 \mathrm{~B}$ & A1 & $0,21 \mathrm{~B}$ & $\mathrm{~S} 2$ & $0,33 \mathrm{~B}$ & S4 & $0,31 \mathrm{C}$ & $\mathrm{S} 2$ & $0,19 \mathrm{C}$ \\
\hline & & & & & & $\mathrm{S} 1$ & $0,30 \mathrm{~B}$ & $\mathrm{~S} 1$ & $0,28 \mathrm{C}$ & S1 & $0,17 \mathrm{C}$ \\
\hline $\mathrm{CV}(\%)$ & 27,48 & & 24,87 & & 24,34 & & 21,90 & & 18,43 & & 24,46 \\
\hline
\end{tabular}

${ }^{1}$ Médias na mesma coluna, seguidas de mesma letra, não diferem entre si, pelo teste de Tukey $(\mathrm{P}<0,05)$. 
De maneira geral, verifica-se para a cultivar Seascape maior acúmulo de biomassa devido à maior capacidade fotossintética no ambiente A3 e no sistema S3 (BUTLER et al., 2002), concordando com os resultados encontrados para a cultivar Campinas.

$\mathrm{Na}$ Tabela 4, em que estão apresentados os desdobramentos para a AF, no período de $\mathrm{MJJ}$ destacaram-se os sistemas S4 e S3, em todos os ambientes. Nos períodos de ASO e NDJFM, o sistema S3, também, apresentou maior AF, nos ambientes A2 e A3, e, no período de NDJFM, esse sistema não diferiu do sistema S4.

No período de MJJ para o sistema S1, os ambientes A2 e A3 não diferiram entre si, apresentando maiores valores de AF promovendo, possivelmente, aumento da taxa fotossintética (PIRES et al., 1999). Para os sistemas S3 e S4 houve destaque para o ambiente A3 (BURIOL et al., 1997; SEGOVIA et al., 1997). No entanto, não se observou diferença entre os ambientes A3 e A1 no sistema S4. No período de ASO para o sistema S1, o ambiente $\mathrm{A} 3$, que não diferiu do ambiente $\mathrm{A} 1$, apresentou maior $\mathrm{AF}$ que o ambiente $\mathrm{A} 2$. No sistema $\mathrm{S} 3$ o ambiente $\mathrm{A} 3$, que não diferiu do ambiente A2, apresentou maior AF que o ambiente A1. O ambiente A3 apresentou maior AF no período de NDJFM, para os sistemas S3 e S4 (Tabela 4).

No período de MJJ nos três ambientes e no período NDJFM destacaram-se os sistemas S3 e S4 para a MFF, e no período de ASO destacaram-se os sistemas S3 e S2, ou seja, nos três períodos o sistema $\mathrm{S} 3$ apresentou destaque para a variável MFF (Tabela 5).

Para o sistema $\mathrm{S} 1$, no primeiro período, o ambiente A3 foi superior ao A1, no segundo período, o ambiente A3 foi superior ao A2. Para o sistema S2, no primeiro período, $\mathrm{o}$ ambiente A3 foi superior ao A1 e, no segundo período, o ambiente A3 superou os demais ambientes. Para o sistema $\mathrm{S} 3$, em todos os períodos, o ambiente A3 apresentou maior valor de MFF, no entanto para o sistema S4 apenas no último período pôde ser observada essa característica. No período 2, já observava-se para esse sistema, que o ambiente A3 estava se destacando, mesmo não diferindo do ambiente 1 (Tabela 5)

Os desdobramentos para a MSF (Tabela 6) mostram, nos diferentes ambientes, que os sistemas S3 e S4 apresentaram maiores valores no período de MJJ. Essa evidência, também pode ser observada no período de NDJFM para os ambientes A2 e A3. No entanto, no período de ASO destacaram-se os sistemas S3 e S2 dentro dos diferentes ambientes. Essa característica marcante de destaque do sistema S3, sistema contendo fibra de coco, concorda com os comentários e resultados encontrados por Nunes (2000), citado por Carrijo et al. (2004).

No sistema S2, no período de ASO, no sistema S3 nos três períodos e no sistema $\mathrm{S} 4$ nos períodos de ASO e

Tabela 4 - Desdobramentos dos sistemas (S) dentro dos ambientes (A) e desdobramentos dos ambientes (A) dentro dos sistemas (S), para a área foliar (AF) $\mathrm{em} \mathrm{mm}^{2}$ da cultivar Seascape, nos períodos de maio a julho (MJJ), agosto a outubro (ASO), novembro a março (NDJFM) ${ }^{1}$.

\begin{tabular}{|c|c|c|c|c|c|c|c|c|c|c|c|c|c|}
\hline \multicolumn{6}{|c|}{ Sistemas (S) dentro dos Ambientes (A) } & \multicolumn{8}{|c|}{ Ambientes (A) dentro dos Sistemas (S) } \\
\hline \multicolumn{14}{|c|}{ MJJ } \\
\hline & A1 & & A2 & & A3 & & S1 & & $\mathrm{S} 2$ & & S3 & & S4 \\
\hline S4 & $98,26 \mathrm{~A}$ & $\mathrm{~S} 4$ & $91,36 \mathrm{~A}$ & S3 & $119,00 \mathrm{~A}$ & $\mathrm{~A} 2$ & $61,64 \mathrm{~A}$ & A3 & $62,57 \mathrm{~A}$ & A3 & $119,00 \mathrm{~A}$ & A3 & $110,87 \mathrm{~A}$ \\
\hline S3 & $83,98 \mathrm{~A}$ & S3 & $81,69 \mathrm{~A}$ & S4 & $110,87 \mathrm{~A}$ & A3 & $61,12 \mathrm{~A}$ & A2 & $57,90 \mathrm{~A}$ & A1 & $83,98 \mathrm{~B}$ & A1 & $98,26 \mathrm{AB}$ \\
\hline $\mathrm{S} 2$ & $48,00 \mathrm{~B}$ & $\mathrm{~S} 1$ & $61,64 \mathrm{~B}$ & $\mathrm{~S} 2$ & $62,57 \mathrm{~B}$ & A1 & 39,68 B & A1 & $48,00 \mathrm{~A}$ & A2 & $81,69 \mathrm{~B}$ & A2 & $91,36 \mathrm{~B}$ \\
\hline $\mathrm{S} 1$ & $39,68 \mathrm{~B}$ & $\mathrm{~S} 2$ & $57,90 \mathrm{~B}$ & S1 & $61,12 \mathrm{~B}$ & & & & & & & & \\
\hline \multicolumn{14}{|c|}{ ASO } \\
\hline \multicolumn{2}{|r|}{ A1 } & \multicolumn{2}{|r|}{$\mathrm{A} 2$} & \multicolumn{2}{|r|}{ A3 } & \multicolumn{2}{|r|}{ S1 } & \multicolumn{2}{|r|}{ S2 } & \multicolumn{2}{|r|}{ S3 } & \multicolumn{2}{|r|}{ S4 } \\
\hline S3 & $59,25 \mathrm{~A}$ & $\mathrm{~S} 3$ & $75,25 \mathrm{~A}$ & S3 & $80,99 \mathrm{~A}$ & A3 & $55,76 \mathrm{~A}$ & A3 & $62,28 \mathrm{~A}$ & A3 & $80,99 \mathrm{~A}$ & A3 & $51,94 \mathrm{~A}$ \\
\hline $\mathrm{S} 2$ & $53,94 \mathrm{~A}$ & $\mathrm{~S} 2$ & $59,80 \mathrm{~B}$ & $\mathrm{~S} 2$ & $62,28 \mathrm{~B}$ & A1 & $49,73 \mathrm{AB}$ & A2 & $59,80 \mathrm{~A}$ & A2 & $75,25 \mathrm{~A}$ & A1 & $50,28 \mathrm{~A}$ \\
\hline $\mathrm{S} 4$ & $50,28 \mathrm{~A}$ & $\mathrm{~S} 4$ & $45,77 \mathrm{C}$ & $\mathrm{S} 1$ & $55,76 \mathrm{~B}$ & A2 & $38,45 \mathrm{~B}$ & A1 & $53,94 \mathrm{~A}$ & A1 & $59,25 \mathrm{~B}$ & A2 & $45,77 \mathrm{~A}$ \\
\hline $\mathrm{S} 1$ & $49,73 \mathrm{~A}$ & $\mathrm{~S} 1$ & $38,45 \mathrm{C}$ & $\mathrm{S} 4$ & $51,94 \mathrm{~B}$ & & & & & & & & \\
\hline \multicolumn{14}{|c|}{ NDJFM } \\
\hline \multicolumn{2}{|r|}{ A1 } & & $\mathrm{A} 2$ & & A3 & \multicolumn{2}{|r|}{ S1 } & \multicolumn{2}{|r|}{ S2 } & \multicolumn{2}{|r|}{ S3 } & \multicolumn{2}{|r|}{ S4 } \\
\hline S3 & $43,25 \mathrm{~A}$ & $\mathrm{~S} 3$ & $55,40 \mathrm{~A}$ & S3 & $71,97 \mathrm{~A}$ & A1 & $38,27 \mathrm{~A}$ & $\mathrm{~A} 2$ & $39,79 \mathrm{~A}$ & A3 & $71,97 \mathrm{~A}$ & A3 & 68 \\
\hline S4 & $38,70 \mathrm{~A}$ & $\mathrm{~S} 2$ & 39,79 B & S4 & $68,32 \mathrm{~A}$ & A3 & $36,52 \mathrm{~A}$ & A1 & $32,86 \mathrm{~A}$ & A2 & $55,40 \mathrm{~B}$ & A2 & $38,78 \mathrm{~B}$ \\
\hline $\mathrm{S} 1$ & $38,27 \mathrm{~A}$ & $\mathrm{~S} 4$ & $38,78 \mathrm{~B}$ & $\mathrm{~S} 1$ & $36,52 \mathrm{~B}$ & A2 & $29,60 \mathrm{~A}$ & A3 & $32,84 \mathrm{~A}$ & A1 & $43,25 \mathrm{C}$ & A1 & $38,70 \mathrm{~B}$ \\
\hline $\mathrm{S} 2$ & $32,86 \mathrm{~A}$ & $\mathrm{~S} 1$ & $29,59 \mathrm{~B}$ & $\mathrm{~S} 2$ & $32,84 \mathrm{~B}$ & & & & & & & & \\
\hline
\end{tabular}

${ }^{1}$ Médias na mesma coluna, seguidas de mesma letra, não diferem entre si, pelo teste de Tukey $(\mathrm{P}<0,05)$. 
Tabela 5 - Desdobramentos dos sistemas (S) dentro dos ambientes (A) e desdobramentos dos ambientes (A) dentro dos sistemas (S), para a massa fresca foliar (MFF) em gramas da cultivar Seascape, nos períodos de maio a julho (MJJ), agosto a outubro (ASO), novembro a março (NDJFM) ${ }^{1}$.

\begin{tabular}{|c|c|c|c|c|c|c|c|c|c|c|c|c|c|}
\hline \multicolumn{6}{|c|}{ Sistemas (S) dentro dos Ambientes (A) } & \multicolumn{8}{|c|}{ Ambientes (A) dentro dos Sistemas (S) } \\
\hline \multicolumn{14}{|c|}{ MJJ } \\
\hline & A1 & & A2 & & A3 & & S1 & & $\mathrm{S} 2$ & & S3 & & $\mathrm{S} 4$ \\
\hline S4 & $2,41 \mathrm{~A}$ & S4 & $2,20 \mathrm{~A}$ & S3 & $3,10 \mathrm{~A}$ & A3 & $1,42 \mathrm{~A}$ & A3 & $1,44 \mathrm{~A}$ & A3 & $3,10 \mathrm{~A}$ & A3 & $2,61 \mathrm{~A}$ \\
\hline S3 & $2,14 \mathrm{~A}$ & $\mathrm{~S} 3$ & $2,07 \mathrm{~A}$ & $\mathrm{~S} 4$ & $2,61 \mathrm{~A}$ & $\mathrm{~A} 2$ & $1,28 \mathrm{AB}$ & $\mathrm{A} 2$ & $1,30 \mathrm{AB}$ & A1 & $2,14 \mathrm{~B}$ & A1 & $2,41 \mathrm{~A}$ \\
\hline S2 & $1,06 \mathrm{~B}$ & $\mathrm{~S} 2$ & $1,30 \mathrm{~B}$ & $\mathrm{~S} 2$ & $1,44 \mathrm{~B}$ & A1 & $0,87 \mathrm{~B}$ & A1 & $1,06 \mathrm{~B}$ & $\mathrm{~A} 2$ & $2,06 \mathrm{~B}$ & $\mathrm{~A} 2$ & $2,20 \mathrm{~A}$ \\
\hline S1 & $0,87 \mathrm{~B}$ & $\mathrm{~S} 1$ & $1,28 \mathrm{~B}$ & $\mathrm{~S} 1$ & $1,42 \mathrm{~B}$ & & & & & & & & \\
\hline \multicolumn{14}{|c|}{ ASO } \\
\hline \multicolumn{2}{|r|}{ A1 } & \multicolumn{2}{|r|}{ A2 } & \multicolumn{2}{|r|}{ A3 } & \multicolumn{2}{|r|}{ S1 } & \multicolumn{2}{|r|}{ S2 } & \multicolumn{2}{|r|}{ S3 } & \multicolumn{2}{|r|}{ S4 } \\
\hline S3 & $1,51 \mathrm{~A}$ & S3 & $1,63 \mathrm{~A}$ & $\mathrm{~S} 3$ & $2,21 \mathrm{~A}$ & A3 & $1,32 \mathrm{~A}$ & A3 & $1,77 \mathrm{~A}$ & A3 & $2,21 \mathrm{~A}$ & A3 & $1,30 \mathrm{~A}$ \\
\hline $\mathrm{S} 2$ & $1,33 \mathrm{AB}$ & $\mathrm{S} 2$ & $1,38 \mathrm{~A}$ & $\mathrm{~S} 2$ & $1,77 \mathrm{~B}$ & A1 & $1,17 \mathrm{AB}$ & $\mathrm{A} 2$ & $1,38 \mathrm{~B}$ & $\mathrm{~A} 2$ & $1,63 \mathrm{~B}$ & A1 & $1,10 \mathrm{AB}$ \\
\hline S1 & $1,17 \mathrm{~B}$ & S4 & $0,96 \mathrm{~B}$ & $\mathrm{~S} 1$ & $1,32 \mathrm{C}$ & $\mathrm{A} 2$ & $0,85 \mathrm{~B}$ & A1 & $1,33 \mathrm{~B}$ & A1 & $1,51 \mathrm{~B}$ & A2 & $0,96 \mathrm{~B}$ \\
\hline S4 & $1,10 \mathrm{~B}$ & $\mathrm{~S} 1$ & $0,85 \mathrm{~B}$ & $\mathrm{~S} 4$ & $1,30 \mathrm{C}$ & & & & & & & & \\
\hline \multicolumn{14}{|c|}{ NDJFM } \\
\hline \multicolumn{2}{|r|}{ A1 } & \multicolumn{2}{|r|}{$\mathrm{A} 2$} & \multicolumn{2}{|r|}{ A3 } & \multicolumn{2}{|r|}{ S1 } & \multicolumn{2}{|r|}{$\mathrm{S} 2$} & \multicolumn{2}{|r|}{ S3 } & \multicolumn{2}{|r|}{ S4 } \\
\hline S3 & $0,94 \mathrm{~A}$ & $\mathrm{~S} 3$ & $1,27 \mathrm{~A}$ & 3 & $1,79 \mathrm{~A}$ & A3 & $0,69 \mathrm{~A}$ & A1 & $0,79 \mathrm{~A}$ & A3 & $1,79 \mathrm{~A}$ & A3 & $1,56 \mathrm{~A}$ \\
\hline S4 & $0,80 \mathrm{AB}$ & $\mathrm{S} 4$ & $0,73 \mathrm{~B}$ & 4 & $1,56 \mathrm{~A}$ & A1 & $0,65 \mathrm{~A}$ & $\mathrm{~A} 2$ & $0,70 \mathrm{~A}$ & $\mathrm{~A} 2$ & $1,27 \mathrm{~B}$ & A1 & $0,80 \mathrm{~B}$ \\
\hline $\mathrm{S} 2$ & $0,79 \mathrm{AB}$ & $\mathrm{S} 2$ & $0,70 \mathrm{~B}$ & 2 & $0,69 \mathrm{~B}$ & $\mathrm{~A} 2$ & $0,48 \mathrm{~A}$ & A3 & $0,69 \mathrm{~A}$ & A1 & $0,94 \mathrm{C}$ & A2 & $0,73 \mathrm{~B}$ \\
\hline S1 & $0,65 \mathrm{~B}$ & $\mathrm{~S} 1$ & $0,48 \mathrm{~B}$ & 1 & $0,69 \mathrm{~B}$ & & & & & & & & \\
\hline
\end{tabular}

${ }^{1}$ Médias na mesma coluna, seguidas de mesma letra, não diferem entre si, pelo teste de Tukey $(\mathrm{P}<0,05)$.

NDJFM destacou-se o ambiente A3 que apresentou maior resultado para a MSF; no entanto, esse ambiente não diferiu do ambiente $\mathrm{A} 2$, no sistema $\mathrm{S} 1 \mathrm{em} \mathrm{MJJ}$ e não diferiu do ambiente A1, em ASO (Tabela 6).

A cultivar Seascape apresentou o mesmo comportamento encontrado para a cultivar Campinas, em relação à diminuição dos valores foliares com o passar dos períodos, sugerindo, novamente, um maior gasto de energia pela planta, devido à uma maior evapotranspiração e, conseqüentemente, menor acúmulo de massa.

Sobre a cultivar Sweet Charlie (Tabelas 7, 8, 9 e 10) observa-se, através da Tabela 7, para a AF que, em todos os períodos, o destaque foi para o ambiente A3, porém nos dois primeiros períodos esse ambiente não diferiu do ambiente A2. O ambiente A3 também apresentou maior resultado para MFF e MSF em todos os períodos, em que o mesmo não diferiu do ambiente $\mathrm{A} 2$, diferindo apenas, no período de MJJ, para a MSF (Tabela 7).

O sistema S3 (Tabela 7) apresentou maiores valores para as variáveis estudadas nos diferentes períodos de coleta, apresentando resultados similares com o sistema S4 apenas para AF, no primeiro e último período de análise. Novamente, os resultados encontrados para o sistema S3, concordam com Carrijo et al. (2002) e Nunes (2000), citados por Carrijo et al. (2004), a respeito da utilização de fibra de coco como substrato, a qual promove um excelente desenvolvimento à planta, principalmente por reter uma maior quantidade de umidade e propiciar um melhor desenvolvimento do sistema radicular, dessa maneira propiciando maior acúmulo de biomassa e maior taxa de fotossíntese (BUTLER et al., 2002).

Nos desdobramentos para a AF, nos ambiente A2 e A3 destacaram-se os sistema S3 e S4 no período de MJJ, nesses ambientes no período de NDJFM destacou-se apenas o sistema S3, enquanto que no período de ASO, apenas no ambiente A2, destacou-se o sistema S3. No último período, os sistemas $\mathrm{S} 3$ e $\mathrm{S} 4$ apresentaram maiores resultados no ambiente $\mathrm{A} 3$, assim como o sistema $\mathrm{S} 2$, no período de ASO (Tabela 8).

$\mathrm{Na}$ Tabela 9, os desdobramentos da MFF destacaram o sistema S3, dentro dos ambientes, e, em alguns casos, esse sistema não diferiu do sistema $S 4$, por exemplo, nos ambientes A2 e A3 do período de MJJ e no ambiente A3, no período de NDJFM. O mesmo também não diferiu do sistema S2, no período de ASO para os ambientes A1 e A3. Para o sistema S4 em MJJ, para os sistemas S1, S2 e S3 em ASO e para os sistemas S3 e S4 em NDJFM, o ambiente A3 apresentou os maiores valores de MFF. Esse ambiente não diferiu do ambiente A2, no sistema S3 em MJJ. 
Tabela 6 - Desdobramentos dos sistemas (S) dentro dos ambientes (A) e desdobramentos dos ambientes (A) dentro dos sistemas (S), para a massa seca foliar (MSF) em gramas da cultivar Seascape, nos períodos de maio a julho (MJJ), agosto a outubro (ASO), novembro a março (NDJFM) ${ }^{1}$.

\begin{tabular}{|c|c|c|c|c|c|c|c|c|c|c|c|c|c|}
\hline \multicolumn{6}{|c|}{ Sistemas (S) dentro dos Ambientes (A) } & \multicolumn{8}{|c|}{ Ambientes (A) dentro dos Sistemas (S) } \\
\hline \multicolumn{14}{|c|}{ MJJ } \\
\hline \multicolumn{2}{|c|}{ A1 } & \multicolumn{2}{|r|}{ A2 } & \multicolumn{2}{|r|}{ A3 } & \multicolumn{2}{|r|}{ S1 } & \multicolumn{2}{|r|}{ S2 } & \multicolumn{2}{|c|}{ S3 } & \multicolumn{2}{|r|}{ S4 } \\
\hline S4 & $0,57 \mathrm{~A}$ & S4 & $0,51 \mathrm{~A}$ & S3 & $0,73 \mathrm{~A}$ & A3 & $0,35 \mathrm{~A}$ & $\mathrm{~A} 2$ & $0,35 \mathrm{~A}$ & A3 & $0,73 \mathrm{~A}$ & A3 & $0,62 \mathrm{~A}$ \\
\hline $\mathrm{S} 3$ & $0,52 \mathrm{~A}$ & S3 & $0,51 \mathrm{~A}$ & $\mathrm{~S} 4$ & $0,62 \mathrm{~B}$ & $\mathrm{~A} 2$ & $0,34 \mathrm{AB}$ & A3 & $0,34 \mathrm{~A}$ & A1 & $0,52 \mathrm{~B}$ & A1 & $0,57 \mathrm{~A}$ \\
\hline $\mathrm{S} 2$ & $0,30 \mathrm{~B}$ & $\mathrm{~S} 2$ & $0,35 \mathrm{~B}$ & S1 & $0,35 \mathrm{C}$ & A1 & $0,23 \mathrm{~B}$ & A1 & $0,30 \mathrm{~A}$ & $\mathrm{~A} 2$ & $0,51 \mathrm{~B}$ & $\mathrm{~A} 2$ & $0,51 \mathrm{~A}$ \\
\hline $\mathrm{S} 1$ & $0,23 \mathrm{~B}$ & $\mathrm{~S} 1$ & $0,34 \mathrm{~B}$ & $\mathrm{~S} 2$ & $0,34 \mathrm{C}$ & & & & & & & & \\
\hline \multicolumn{14}{|c|}{ ASO } \\
\hline \multicolumn{2}{|c|}{ A1 } & \multicolumn{2}{|r|}{ A2 } & \multicolumn{2}{|r|}{ A3 } & & $\mathrm{S} 1$ & \multicolumn{2}{|r|}{$\mathrm{S} 2$} & \multicolumn{2}{|r|}{ S3 } & \multicolumn{2}{|r|}{ S4 } \\
\hline $\mathrm{S} 3$ & $0,37 \mathrm{~A}$ & S3 & $0,41 \mathrm{~A}$ & $\mathrm{~S} 3$ & $0,54 \mathrm{~A}$ & A3 & $0,330 \mathrm{~A}$ & A3 & $0,45 \mathrm{~A}$ & A3 & $0,54 \mathrm{~A}$ & A3 & $0,39 \mathrm{~A}$ \\
\hline $\mathrm{S} 2$ & $0,33 \mathrm{AB}$ & $\mathrm{S} 2$ & $0,34 \mathrm{~A}$ & $\mathrm{~S} 2$ & $0,45 \mathrm{~B}$ & $\mathrm{~A} 1$ & $0,29 \mathrm{~A}$ & $\mathrm{~A} 2$ & $0,34 \mathrm{~B}$ & $\mathrm{~A} 2$ & $0,41 \mathrm{~B}$ & A1 & $0,28 \mathrm{~B}$ \\
\hline S1 & $0,29 \mathrm{AB}$ & S4 & $0,25 \mathrm{~B}$ & $\mathrm{~S} 4$ & $0,39 \mathrm{BC}$ & $\mathrm{A} 2$ & $0,21 \mathrm{~B}$ & A1 & $0,33 \mathrm{~B}$ & A1 & $0,37 \mathrm{~B}$ & $\mathrm{~A} 2$ & $0,25 \mathrm{~B}$ \\
\hline S4 & $0,28 \mathrm{~B}$ & $\mathrm{~S} 1$ & $0,21 \mathrm{~B}$ & $\mathrm{~S} 1$ & $0,33 \mathrm{C}$ & & & & & & & & \\
\hline \multicolumn{14}{|c|}{ NDJFM } \\
\hline \multicolumn{2}{|c|}{ A1 } & \multicolumn{2}{|r|}{$\mathrm{A} 2$} & \multicolumn{2}{|r|}{ A3 } & \multicolumn{2}{|r|}{ S1 } & \multicolumn{2}{|r|}{$\mathrm{S} 2$} & \multicolumn{2}{|r|}{ S3 } & \multicolumn{2}{|r|}{ S4 } \\
\hline $\mathrm{S} 3$ & $0,23 \mathrm{~A}$ & S3 & $0,38 \mathrm{~A}$ & S3 & $0,55 \mathrm{~A}$ & A3 & $0,19 \mathrm{~A}$ & A1 & $0,22 \mathrm{~A}$ & A3 & 0,5 & A3 & $0,44 \mathrm{~A}$ \\
\hline $\mathrm{S} 2$ & $0,22 \mathrm{~A}$ & $\mathrm{~S} 4$ & $0,20 \mathrm{~B}$ & $\mathrm{~S} 4$ & $0,44 \mathrm{~B}$ & $\mathrm{~A} 1$ & $0,19 \mathrm{~A}$ & $\mathrm{~A} 2$ & $0,18 \mathrm{~A}$ & $\mathrm{~A} 2$ & $0,38 \mathrm{~B}$ & A1 & $0,21 \mathrm{~B}$ \\
\hline $\mathrm{S} 4$ & $0,21 \mathrm{~A}$ & $\mathrm{~S} 2$ & $0,18 \mathrm{~B}$ & $\mathrm{~S} 1$ & $0,19 \mathrm{C}$ & $\mathrm{A} 2$ & $0,14 \mathrm{~A}$ & A3 & $0,16 \mathrm{~A}$ & A1 & $0,23 \mathrm{C}$ & $\mathrm{A} 2$ & $0,20 \mathrm{~B}$ \\
\hline S1 & $0,19 \mathrm{~A}$ & $\mathrm{~S} 1$ & $0,14 \mathrm{~B}$ & $\mathrm{~S} 2$ & $0,16 \mathrm{C}$ & & & & & & & & \\
\hline
\end{tabular}

${ }^{1}$ Médias na mesma coluna, seguidas de mesma letra, não diferem entre si, pelo teste de Tukey $(\mathrm{P}<0,05)$.

Tabela 7 - Área foliar (AF), massa fresca foliar (MFF) e massa seca foliar (MSF) para a cultivar Sweet Charlie nos ambientes (A) e nos Sistemas (S), nos períodos de maio a julho (MJJ), agosto a outubro (ASO), novembro a março (NDJFM) ${ }^{1}$.

\begin{tabular}{|c|c|c|c|c|c|c|c|c|c|c|c|}
\hline \multicolumn{8}{|c|}{ Ambientes (A) } & \multicolumn{4}{|c|}{ Sistemas (S) } \\
\hline \multicolumn{12}{|c|}{ Área Foliar (AF) $\left(\mathrm{mm}^{2}\right)$} \\
\hline \multicolumn{2}{|c|}{ MJJ } & \multicolumn{2}{|r|}{ ASO } & \multicolumn{2}{|c|}{ NDJFM } & \multicolumn{2}{|c|}{ MJJ } & \multicolumn{2}{|c|}{ ASO } & \multicolumn{2}{|c|}{ NDJFM } \\
\hline A3 & $75,49 \mathrm{~A}$ & A3 & $51,85 \mathrm{~A}$ & A3 & $47,97 \mathrm{~A}$ & S4 & $83,82 \mathrm{~A}$ & S3 & $56,83 \mathrm{~A}$ & S3 & $49,98 \mathrm{~A}$ \\
\hline $\mathrm{A} 2$ & $69,92 \mathrm{~A}$ & $\mathrm{~A} 2$ & $46,92 \mathrm{AB}$ & $\mathrm{A} 2$ & $33,31 \mathrm{~B}$ & S3 & $82,69 \mathrm{~A}$ & $\mathrm{~S} 2$ & $47,77 \mathrm{~B}$ & S4 & $47,05 \mathrm{~A}$ \\
\hline \multirow[t]{2}{*}{ A1 } & $57,63 \mathrm{~B}$ & A1 & $41,58 \mathrm{~B}$ & A1 & $32,45 \mathrm{~B}$ & $\mathrm{~S} 2$ & $56,21 \mathrm{~B}$ & S1 & $45,03 \mathrm{~B}$ & S2 & $27,81 \mathrm{~B}$ \\
\hline & & & & & & $\mathrm{S} 1$ & $48,00 \mathrm{C}$ & S4 & $37,51 \mathrm{C}$ & S1 & $26,79 \mathrm{~B}$ \\
\hline $\mathrm{CV}(\%)$ & 20,85 & & 20,82 & & 21,69 & & 16,83 & & 18,62 & & 23,94 \\
\hline \multicolumn{12}{|c|}{ Massa Fresca Foliar (MFF) (g) } \\
\hline \multicolumn{2}{|c|}{ MJJ } & \multicolumn{2}{|r|}{ ASO } & \multicolumn{2}{|c|}{ NDJFM } & \multicolumn{2}{|c|}{ MJJ } & \multicolumn{2}{|c|}{ ASO } & \multicolumn{2}{|c|}{ NDJFM } \\
\hline A3 & $1,71 \mathrm{~A}$ & A3 & $1,19 \mathrm{~A}$ & A3 & $1,06 \mathrm{~A}$ & $\mathrm{~S} 3$ & $1,92 \mathrm{~A}$ & S3 & $1,32 \mathrm{~A}$ & S3 & $1,15 \mathrm{~A}$ \\
\hline $\mathrm{A} 2$ & $1,50 \mathrm{~B}$ & $\mathrm{~A} 2$ & $0,98 \mathrm{~B}$ & $\mathrm{~A} 2$ & $0,69 \mathrm{~B}$ & S4 & $1,73 \mathrm{~B}$ & $\mathrm{~S} 2$ & $1,06 \mathrm{~B}$ & S4 & $0,98 \mathrm{~B}$ \\
\hline \multirow[t]{2}{*}{ A1 } & $1,25 \mathrm{C}$ & A1 & $0,89 \mathrm{~B}$ & A1 & $0,65 \mathrm{~B}$ & $\mathrm{~S} 2$ & $1,23 \mathrm{C}$ & S1 & $0,90 \mathrm{C}$ & S2 & $0,56 \mathrm{C}$ \\
\hline & & & & & & S1 & $1,06 \mathrm{C}$ & S4 & $0,80 \mathrm{C}$ & S1 & $0,52 \mathrm{C}$ \\
\hline $\mathrm{CV}(\%)$ & 20,91 & & 20,22 & & 23,53 & & 16,83 & & 18,79 & & 25,12 \\
\hline \multicolumn{12}{|c|}{ Massa Seca Foliar (MSF) (g) } \\
\hline \multicolumn{2}{|c|}{ MJJ } & \multicolumn{2}{|r|}{ ASO } & \multicolumn{2}{|c|}{ NDJFM } & \multicolumn{2}{|c|}{$\mathrm{MJJ}$} & \multicolumn{2}{|c|}{$\mathrm{ASO}$} & \multicolumn{2}{|c|}{ NDJFM } \\
\hline A3 & $0,45 \mathrm{~A}$ & A3 & $0,31 \mathrm{~A}$ & A3 & $0,30 \mathrm{~A}$ & S3 & $0,47 \mathrm{~A}$ & S3 & $0,34 \mathrm{~A}$ & S3 & $0,32 \mathrm{~A}$ \\
\hline $\mathrm{A} 2$ & $0,37 \mathrm{~A}$ & A2 & $0,25 \mathrm{~B}$ & $\mathrm{~A} 2$ & $0,20 \mathrm{~B}$ & S4 & $0,38 \mathrm{~B}$ & $\mathrm{~S} 2$ & $0,27 \mathrm{~B}$ & S4 & $0,25 \mathrm{~B}$ \\
\hline \multirow[t]{2}{*}{ A1 } & $0,31 \mathrm{~B}$ & A1 & $0,22 \mathrm{C}$ & A1 & $0,17 \mathrm{C}$ & $\mathrm{S} 2$ & $0,32 \mathrm{C}$ & S1 & $0,22 \mathrm{C}$ & S2 & $0,17 \mathrm{C}$ \\
\hline & & & & & & $\mathrm{S} 1$ & $0,28 \mathrm{C}$ & S4 & $0,22 \mathrm{C}$ & S1 & $0,16 \mathrm{C}$ \\
\hline $\mathrm{CV}(\%)$ & 21,09 & & 20,48 & & 22,30 & & 16,95 & & 18,79 & & 24,20 \\
\hline
\end{tabular}

${ }^{1}$ Médias na mesma coluna, seguidas de mesma letra, não diferem entre si, pelo teste de Tukey $(\mathrm{P}<0,05)$. 
Tabela 8 - Desdobramentos dos sistemas (S) dentro dos ambientes (A) e desdobramentos dos ambientes (A) dentro dos sistemas (S), para a área foliar (AF) em $\mathrm{mm}^{2}$ da cultivar Sweet Charlie, nos períodos de maio a julho (MJJ), agosto a outubro (ASO), novembro a março (NDJFM) ${ }^{1}$.

\begin{tabular}{|c|c|c|c|c|c|c|c|c|c|c|c|c|}
\hline \multicolumn{6}{|c|}{ Sistemas (S) dentro dos Ambientes (A) } & \multicolumn{7}{|c|}{ Ambientes (A) dentro dos Sistemas (S) } \\
\hline \multicolumn{13}{|c|}{ MJJ } \\
\hline & A1 & & $\mathrm{A} 2$ & & A3 & & S1 & & S2 & S3 & & $\mathrm{S} 4$ \\
\hline S3 & $73,66 \mathrm{~A}$ & $\mathrm{~S} 4$ & $88,79 \mathrm{~A}$ & S4 & $99,48 \mathrm{~A}$ & A3 & $52,87 \mathrm{~A}$ & A3 & 60,59 A A3 & $89,01 \mathrm{~A}$ & A3 & $99,48 \mathrm{~A}$ \\
\hline S4 & $63,18 \mathrm{AB}$ & S3 & $85,38 \mathrm{~A}$ & S3 & $89,01 \mathrm{~A}$ & $\mathrm{~A} 2$ & $49,78 \mathrm{~A}$ & $\mathrm{~A} 2$ & $55,72 \mathrm{~A} \quad \mathrm{~A} 2$ & $85,38 \mathrm{AB}$ & $\mathrm{A} 2$ & $88,79 \mathrm{~A}$ \\
\hline S2 & $52,32 \mathrm{BC}$ & $\mathrm{S} 2$ & $55,72 \mathrm{~B}$ & $\mathrm{~S} 2$ & $60,59 \mathrm{~B}$ & A1 & $41,34 \mathrm{~A}$ & A1 & 52,32 A A1 & $73,66 \mathrm{~B}$ & A1 & $63,18 \mathrm{~B}$ \\
\hline S1 & $41,34 \mathrm{C}$ & $\mathrm{S} 1$ & $49,78 \mathrm{~B}$ & $\mathrm{~S} 1$ & $52,87 \mathrm{~B}$ & & & & & & & \\
\hline \multicolumn{13}{|c|}{ ASO } \\
\hline & A1 & & $\mathrm{A} 2$ & & A3 & & $\mathrm{S} 1$ & & S2 & S3 & & $\mathrm{S} 4$ \\
\hline S3 & $52,97 \mathrm{~A}$ & S3 & $58,30 \mathrm{~A}$ & S3 & $59,20 \mathrm{~A}$ & A3 & $52,12 \mathrm{~A}$ & A3 & 58,26 A A3 & $59,20 \mathrm{~A}$ & $\mathrm{~A} 2$ & $2 \mathrm{~A}$ \\
\hline S2 & $42,82 \mathrm{AB}$ & $\mathrm{S} 1$ & $44,84 \mathrm{~B}$ & $\mathrm{~S} 2$ & $58,26 \mathrm{~A}$ & $\mathrm{~A} 2$ & $44,84 \mathrm{AB}$ & A1 & $42,82 \mathrm{~B} \quad \mathrm{~A} 2$ & $58,30 \mathrm{~A}$ & A3 & $37,83 \mathrm{AB}$ \\
\hline S1 & $38,13 \mathrm{BC}$ & $\mathrm{S} 4$ & $42,32 \mathrm{~B}$ & $\mathrm{~S} 1$ & $52,12 \mathrm{~A}$ & A1 & $38,13 \mathrm{~B}$ & A2 & $42,24 \mathrm{~B}$ A1 & $52,97 \mathrm{~A}$ & A1 & $32,39 \mathrm{~B}$ \\
\hline S4 & $32,39 \mathrm{C}$ & $\mathrm{S} 2$ & $42,24 \mathrm{~B}$ & $\mathrm{~S} 4$ & $37,83 \mathrm{~B}$ & & & & & & & \\
\hline \multicolumn{13}{|c|}{ NDJFM } \\
\hline & A1 & & $\mathrm{A} 2$ & & A3 & & $\mathrm{S} 1$ & & $\mathrm{~S} 2$ & S3 & & $\mathrm{S} 4$ \\
\hline S3 & 42,4 & $\mathrm{~S} 3$ & $45,85 \mathrm{~A}$ & S4 & $73,73 \mathrm{~A}$ & $\mathrm{~A} 2$ & 29,2 & A3 & $31,42 \mathrm{~A} \quad \mathrm{~A} 3$ & 61, & A3 & 73 \\
\hline S4 & $35,18 \mathrm{AB}$ & $\mathrm{S} 4$ & $32,24 \mathrm{~B}$ & S3 & $61,64 \mathrm{~B}$ & A1 & $26,03 \mathrm{~A}$ & A1 & $26,14 \mathrm{~A} \quad \mathrm{~A} 2$ & 45 & A1 & $35,18 \mathrm{~B}$ \\
\hline S2 & $26,14 \mathrm{~B}$ & $\mathrm{~S} 1$ & $29,26 \mathrm{~B}$ & $\mathrm{~S} 2$ & $31,42 \mathrm{C}$ & A3 & $25,09 \mathrm{~A}$ & A2 & $25,88 \mathrm{~A} \quad \mathrm{~A} 1$ & $42,44 \mathrm{~B}$ & $\mathrm{~A} 2$ & $32,24 \mathrm{~B}$ \\
\hline S1 & $26,03 \mathrm{~B}$ & $\mathrm{~S} 2$ & $25,88 \mathrm{~B}$ & $\mathrm{~S} 1$ & $25,09 \mathrm{C}$ & & & & & & & \\
\hline
\end{tabular}

${ }^{1}$ Médias na mesma coluna, seguidas de mesma letra, não diferem entre si, pelo teste de Tukey $(\mathrm{P}<0,05)$.

Tabela 9 - Desdobramentos dos sistemas (S) dentro dos ambientes (A) e desdobramentos dos ambientes (A) dentro dos sistemas (S), para a massa fresca foliar (MFF), em gramas, da cultivar Sweet Charlie, nos períodos de maio a julho (MJJ), agosto a outubro (ASO), novembro a março (NDJFM) ${ }^{1}$.

\begin{tabular}{|c|c|c|c|c|c|c|c|c|c|c|c|c|c|}
\hline \multicolumn{6}{|c|}{ Sistemas (S) dentro dos Ambientes (A) } & \multicolumn{8}{|c|}{ Ambientes (A) dentro dos Sistemas (S) } \\
\hline \multicolumn{14}{|c|}{ - } \\
\hline \multicolumn{2}{|c|}{ A1 } & \multicolumn{2}{|r|}{ A2 } & \multicolumn{2}{|r|}{ A3 } & \multicolumn{2}{|r|}{ S1 } & \multicolumn{2}{|r|}{ S2 } & \multicolumn{2}{|r|}{ S3 } & \multicolumn{2}{|r|}{ S4 } \\
\hline S3 & $1,64 \mathrm{~A}$ & S3 & $1,95 \mathrm{~A}$ & S3 & $2,16 \mathrm{~A}$ & A3 & $1,15 \mathrm{~A}$ & A3 & $1,39 \mathrm{~A}$ & A3 & $2,16 \mathrm{~A}$ & A3 & $2,120 \mathrm{~A}$ \\
\hline S4 & $1,31 \mathrm{~B}$ & S4 & $1,75 \mathrm{~A}$ & S4 & $2,12 \mathrm{~A}$ & $\mathrm{~A} 2$ & $1,12 \mathrm{~A}$ & $\mathrm{~A} 2$ & $1,16 \mathrm{~A}$ & $\mathrm{~A} 2$ & $1,95 \mathrm{~A}$ & $\mathrm{~A} 2$ & $1,75 \mathrm{~B}$ \\
\hline $\mathrm{S} 2$ & $1,14 \mathrm{BC}$ & $\mathrm{S} 2$ & $1,16 \mathrm{~B}$ & S2 & $1,39 \mathrm{~B}$ & A1 & $0,91 \mathrm{~A}$ & A1 & $1,14 \mathrm{~A}$ & A1 & $1,64 \mathrm{~B}$ & A1 & $1,31 \mathrm{C}$ \\
\hline $\mathrm{S} 1$ & $0,91 \mathrm{C}$ & $\mathrm{S} 1$ & $1,12 \mathrm{~B}$ & S1 & $1,15 \mathrm{~B}$ & & & & & & & & \\
\hline \multicolumn{14}{|c|}{ ASO } \\
\hline \multicolumn{2}{|c|}{ A1 } & \multicolumn{2}{|r|}{$\mathrm{A} 2$} & \multicolumn{2}{|r|}{ A3 } & \multicolumn{2}{|r|}{ S1 } & \multicolumn{2}{|r|}{$\mathrm{S} 2$} & \multicolumn{2}{|r|}{ S3 } & \multicolumn{2}{|r|}{ S4 } \\
\hline S3 & $1,12 \mathrm{~A}$ & S3 & $1,30 \mathrm{~A}$ & S3 & $1,54 \mathrm{~A}$ & A3 & $1,01 \mathrm{~A}$ & A3 & $1,36 \mathrm{~A}$ & A3 & $1,54 \mathrm{~A}$ & A3 & $0,85 \mathrm{~A}$ \\
\hline $\mathrm{S} 2$ & $0,94 \mathrm{AB}$ & $\mathrm{S} 1$ & $0,93 \mathrm{~B}$ & $\mathrm{~S} 2$ & $1,36 \mathrm{~A}$ & $\mathrm{~A} 2$ & 0,93 B & $\mathrm{A} 1$ & $0,94 \mathrm{~B}$ & A2 & $1,30 \mathrm{~B}$ & A2 & $0,81 \mathrm{~A}$ \\
\hline $\mathrm{S} 1$ & $0,77 \mathrm{~B}$ & $\mathrm{~S} 2$ & $0,88 \mathrm{~B}$ & $\mathrm{~S} 1$ & $1,01 \mathrm{~B}$ & $\mathrm{~A} 1$ & $0,77 \mathrm{~B}$ & $\mathrm{~A} 2$ & $0,88 \mathrm{~B}$ & A1 & $1,12 \mathrm{~B}$ & A1 & $0,74 \mathrm{~A}$ \\
\hline $\mathrm{S} 4$ & $0,74 \mathrm{~B}$ & $\mathrm{~S} 4$ & $0,81 \mathrm{~B}$ & $\mathrm{~S} 4$ & $0,85 \mathrm{~B}$ & & & & & & & & \\
\hline \multicolumn{14}{|c|}{ NDJFM } \\
\hline \multicolumn{2}{|c|}{ A1 } & \multicolumn{2}{|r|}{$\mathrm{A} 2$} & \multicolumn{2}{|r|}{ A3 } & \multicolumn{2}{|r|}{ S1 } & \multicolumn{2}{|r|}{ S2 } & \multicolumn{2}{|r|}{ S3 } & \multicolumn{2}{|r|}{$\mathrm{S} 4$} \\
\hline S3 & $0,96 \mathrm{~A}$ & S3 & $1,02 \mathrm{~A}$ & S4 & $1,62 \mathrm{~A}$ & $\mathrm{~A} 2$ & $0,63 \mathrm{~A}$ & A3 & $0,67 \mathrm{~A}$ & A3 & $1,47 \mathrm{~A}$ & A3 & $1,62 \mathrm{~A}$ \\
\hline S4 & $0,69 \mathrm{~B}$ & $\mathrm{~S} 1$ & $0,63 \mathrm{~B}$ & S3 & $1,47 \mathrm{~A}$ & A3 & $0,47 \mathrm{~A}$ & A1 & $0,50 \mathrm{~A}$ & A2 & $1,02 \mathrm{~B}$ & A1 & $0,69 \mathrm{~B}$ \\
\hline $\mathrm{S} 2$ & $0,50 \mathrm{BC}$ & $\mathrm{S} 4$ & $0,62 \mathrm{~B}$ & $\mathrm{~S} 2$ & $0,67 \mathrm{~B}$ & $\mathrm{~A} 1$ & $0,45 \mathrm{~A}$ & $\mathrm{~A} 2$ & $0,50 \mathrm{~A}$ & A1 & $0,96 \mathrm{~B}$ & A2 & $0,62 \mathrm{~B}$ \\
\hline $\mathrm{S} 1$ & $0,45 \mathrm{C}$ & $\mathrm{S} 2$ & $0,50 \mathrm{~B}$ & $\mathrm{~S} 1$ & $0,47 \mathrm{~B}$ & & & & & & & & \\
\hline
\end{tabular}

${ }^{1}$ Médias na mesma coluna, seguidas de mesma letra, não diferem entre si, pelo teste de Tukey $(\mathrm{P}<0,05)$. 
Nos desdobramentos da MSF, destacou-se o sistema S3, dentro dos ambientes, e, em alguns casos, esse sistema não diferiu do sistema $\mathrm{S} 4$, por exemplo no ambiente A3 dos períodos de MJJ e de NDJFM, e no ambiente A1, do período de NDJFM. O mesmo também não diferiu do sistema S2, no período de ASO, para os ambientes A1 e A3. Para os sistemas S2 e S3, em ASO, e para os sistemas S2, S3 e S4 em NDJFM, o ambiente A3 apresentou os maiores valores de MSF. Nos sistemas S1, S3 e S4 em MJJ, e no sistema S1 em ASO, esse ambiente não diferiu do ambiente A2 (Tabela 10).

As análises foliares para a cultivar Tudla foram realizadas nos dois últimos períodos, ou seja, no período de ASO e NDJFM, devido a problemas fitossanitários (presença de lagarta-rosca) ocorridos com essa cultivar, no período de MJJ (Tabelas 11 e 12). Pela Tabela 11, observa-se, assim como para as demais cultivares de morangueiro estudadas, que o ambiente A3 e o sistema S3 apresentaram os maiores valores de AF, MFF e MSF, e em alguns casos, como por exemplo, AF e MFF, no período de NDJFM, o sistema S3 não diferiu do sistema S4.

Avaliando-se os desdobramentos de AF, MFF e MSF (Tabela 12), da cultivar Tudla para o último período de análise, verifica-se a predominância dos sistemas S3 e S4 em apresentarem maiores resultados para as variáveis foliares, assim como o ambiente A3.

No período de ASO, os desdobramentos dos sistemas (S) dentro dos ambientes (A) e desdobramentos dos ambientes (A) dentro dos sistemas (S), para AF, MFF e MSF, da cultivar Tudla, não foram significativos.

Dentro de ambientes protegidos coberto com filme de polietileno, à medida que a temperatura do ar aumenta em função do aumento da disponibilidade energética na superfície do solo (radiação solar global), a umidade relativa do ar diminui em função do aquecimento do ar (COSTA et al., 2004), dessa forma ocorre a inibição do processo convectivo devido à barreira imposta pela presença do filme plástico no ambiente protegido (CUNHA \& ESCOBEDO, 2003), provocando o efeito estufa. Nesse processo, pode ocorrer uma maior evapotranspiração em função de um maior gasto de energia e, conseqüentemente, acarretar num menor desenvolvimento das plantas. Essa característica pode ter sido mais intensa nos ambientes não climatizados $\mathrm{A} 1$ e $\mathrm{A} 2$, pois no ambiente $\mathrm{A} 3$ o resfriamento evaporativo do ar promovia o aumento da umidade relativa.

Tabela 10 - Desdobramentos dos sistemas (S) dentro dos ambientes (A) e desdobramentos dos ambientes (A) dentro dos sistemas (S), para a massa seca foliar (MSF), em gramas, da cultivar Sweet Charlie, nos períodos de maio a julho (MJJ), agosto a outubro (ASO), novembro a março (NDJFM) ${ }^{1}$.

\begin{tabular}{|c|c|c|c|c|c|c|c|c|c|c|c|c|c|}
\hline & \multicolumn{5}{|c|}{ Sistemas (S) dentro dos Ambientes (A) } & \multicolumn{8}{|c|}{ Ambientes (A) dentro dos Sistemas (S) } \\
\hline \multicolumn{14}{|c|}{ MJJ } \\
\hline & A1 & & A2 & & A3 & & S1 & & $\mathrm{S} 2$ & & S3 & & S4 \\
\hline S3 & $0,40 \mathrm{~A}$ & S3 & $0,51 \mathrm{~A}$ & S3 & $0,51 \mathrm{~A}$ & A3 & $0,31 \mathrm{~A}$ & A3 & $0,35 \mathrm{~A}$ & A3 & $0,51 \mathrm{~A}$ & A3 & $0,45 \mathrm{~A}$ \\
\hline $\mathrm{S} 2$ & $0,31 \mathrm{~B}$ & $\mathrm{~S} 4$ & $0,40 \mathrm{~B}$ & S4 & $0,45 \mathrm{~A}$ & A2 & $0,29 \mathrm{AB}$ & A1 & $0,31 \mathrm{~A}$ & A2 & $0,51 \mathrm{~A}$ & A2 & $0,40 \mathrm{~A}$ \\
\hline S4 & $0,29 \mathrm{BC}$ & $\mathrm{S} 2$ & $0,30 \mathrm{C}$ & $\mathrm{S} 2$ & $0,35 \mathrm{~B}$ & A1 & $0,24 \mathrm{~B}$ & A2 & $0,30 \mathrm{~A}$ & A1 & $0,40 \mathrm{~B}$ & A1 & $0,29 \mathrm{~B}$ \\
\hline S1 & $0,24 \mathrm{C}$ & $\mathrm{S} 1$ & $0,29 \mathrm{C}$ & $\mathrm{S} 1$ & $0,31 \mathrm{~B}$ & & & & & & & & \\
\hline \multicolumn{14}{|c|}{ ASO } \\
\hline & A1 & & $\mathrm{A} 2$ & & A3 & & S1 & & S2 & & S3 & & S4 \\
\hline S3 & $0,27 \mathrm{~A}$ & S3 & $0,34 \mathrm{~A}$ & S3 & $0,40 \mathrm{~A}$ & A3 & $0,24 \mathrm{~A}$ & A3 & $0,35 \mathrm{~A}$ & A3 & $0,40 \mathrm{~A}$ & A3 & $0,24 \mathrm{~A}$ \\
\hline $\mathrm{S} 2$ & $0,24 \mathrm{AB}$ & $\mathrm{S} 2$ & $0,23 \mathrm{~B}$ & $\mathrm{~S} 2$ & $0,35 \mathrm{~A}$ & A2 & $0,23 \mathrm{AB}$ & A1 & $0,24 \mathrm{~B}$ & A2 & $0,34 \mathrm{~B}$ & A2 & $0,22 \mathrm{~A}$ \\
\hline S4 & $0,20 \mathrm{BC}$ & $\mathrm{S} 1$ & $0,23 \mathrm{~B}$ & S1 & $0,24 \mathrm{~B}$ & A1 & $0,18 \mathrm{~B}$ & A2 & $0,23 \mathrm{~B}$ & A1 & $0,27 \mathrm{C}$ & A1 & $0,20 \mathrm{~A}$ \\
\hline S1 & $0,18 \mathrm{C}$ & $\mathrm{S} 4$ & $0,22 \mathrm{~B}$ & S4 & $0,24 \mathrm{~B}$ & & & & & & & & \\
\hline \multicolumn{14}{|c|}{ NDJFM } \\
\hline & A1 & & A2 & & A3 & & S1 & & $\mathrm{S} 2$ & & S3 & & S4 \\
\hline S3 & $0,23 \mathrm{~A}$ & S3 & $0,30 \mathrm{~A}$ & S3 & $0,43 \mathrm{~A}$ & A2 & $0,19 \mathrm{~A}$ & A3 & $0,22 \mathrm{~A}$ & A3 & $0,43 \mathrm{~A}$ & A3 & $0,41 \mathrm{~A}$ \\
\hline S4 & $0,18 \mathrm{AB}$ & $\mathrm{S} 1$ & $0,19 \mathrm{~B}$ & $\mathrm{~S} 4$ & $0,41 \mathrm{~A}$ & A3 & $0,15 \mathrm{~A}$ & A1 & $0,14 \mathrm{~B}$ & $\mathrm{~A} 2$ & $0,30 \mathrm{~B}$ & A1 & $0,18 \mathrm{~B}$ \\
\hline $\mathrm{S} 2$ & $0,14 \mathrm{~B}$ & $\mathrm{~S} 4$ & $0,18 \mathrm{~B}$ & $\mathrm{~S} 2$ & $0,22 \mathrm{~B}$ & A1 & $0,14 \mathrm{~A}$ & $\mathrm{~A} 2$ & $0,14 \mathrm{~B}$ & A1 & $0,23 \mathrm{C}$ & $\mathrm{A} 2$ & $0,18 \mathrm{~B}$ \\
\hline S1 & $0,14 \mathrm{~B}$ & $\mathrm{~S} 2$ & $0,14 \mathrm{~B}$ & $\mathrm{~S} 1$ & $0,15 \mathrm{C}$ & & & & & & & & \\
\hline
\end{tabular}

${ }^{1}$ Médias na mesma coluna, seguidas de mesma letra, não diferem entre si, pelo teste de Tukey $(\mathrm{P}<0,05)$. 
Tabela 11 - Área foliar (AF), massa fresca foliar (MFF) e massa seca foliar (MSF) para a cultivar Tudla nos ambientes (A) e nos Sistemas (S), nos períodos de agosto a outubro (ASO), novembro a março (NDJFM) ${ }^{1}$.

\begin{tabular}{|c|c|c|c|c|c|c|c|}
\hline \multicolumn{4}{|c|}{ Ambientes (A) } & \multicolumn{4}{|c|}{ Sistemas (S) } \\
\hline \multicolumn{2}{|c|}{ ASO } & \multicolumn{2}{|c|}{ NDJFM } & \multicolumn{2}{|c|}{ ASO } & \multicolumn{2}{|c|}{ NDJFM } \\
\hline A3 & $66,78 \mathrm{~A}$ & A3 & $51,81 \mathrm{~A}$ & S3 & $70,08 \mathrm{~A}$ & S4 & $54,77 \mathrm{~A}$ \\
\hline A1 & $53,72 \mathrm{~B}$ & $\mathrm{~A} 2$ & $37,94 \mathrm{~B}$ & $\mathrm{~S} 1$ & $58,74 \mathrm{~B}$ & S3 & $51,89 \mathrm{~A}$ \\
\hline \multirow[t]{2}{*}{$\mathrm{A} 2$} & $50,14 \mathrm{~B}$ & A1 & $35,51 \mathrm{~B}$ & $\mathrm{~S} 2$ & $57,77 \mathrm{~B}$ & $\mathrm{~S} 1$ & $30,89 \mathrm{~B}$ \\
\hline & & & & S4 & $40,92 \mathrm{C}$ & $\mathrm{S} 2$ & 29,46 B \\
\hline $\mathrm{CV}(\%)$ & 17,91 & & 29,07 & & 21,45 & & 24,63 \\
\hline \multicolumn{8}{|c|}{ Massa Fresca Foliar (MFF) (g) } \\
\hline \multicolumn{2}{|c|}{$\mathrm{ASO}$} & \multicolumn{2}{|c|}{ NDJFM } & \multicolumn{2}{|c|}{ ASO } & \multicolumn{2}{|c|}{ NDJFM } \\
\hline A3 & $1,71 \mathrm{~A}$ & A3 & $1,16 \mathrm{~A}$ & S3 & $1,68 \mathrm{~A}$ & S3 & $1,22 \mathrm{~A}$ \\
\hline A1 & $1,21 \mathrm{~B}$ & $\mathrm{~A} 2$ & $0,83 \mathrm{~B}$ & $\mathrm{~S} 1$ & $1,44 \mathrm{~B}$ & S4 & $1,16 \mathrm{~A}$ \\
\hline \multirow[t]{2}{*}{$\mathrm{A} 2$} & $1,19 \mathrm{~B}$ & A1 & $0,76 \mathrm{~B}$ & $\mathrm{~S} 2$ & $1,42 \mathrm{~B}$ & $\mathrm{~S} 2$ & $0,65 \mathrm{~B}$ \\
\hline & & & & S4 & $0,95 \mathrm{C}$ & $\mathrm{S} 1$ & $0,63 \mathrm{~B}$ \\
\hline $\mathrm{CV}(\%)$ & 19,02 & & 30,36 & & 20,93 & & 24,49 \\
\hline \multicolumn{8}{|c|}{ Massa Seca Foliar (MSF) (g) } \\
\hline \multicolumn{2}{|c|}{$\mathrm{ASO}$} & \multicolumn{2}{|c|}{ NDJFM } & \multicolumn{2}{|c|}{ ASO } & \multicolumn{2}{|c|}{ NDJFM } \\
\hline A3 & $0,41 \mathrm{~A}$ & A3 & $0,30 \mathrm{~A}$ & S3 & $0,40 \mathrm{~A}$ & S3 & $0,32 \mathrm{~A}$ \\
\hline A1 & $0,29 \mathrm{~B}$ & $\mathrm{~A} 2$ & $0,22 \mathrm{~B}$ & $\mathrm{~S} 2$ & $0,35 \mathrm{~B}$ & S4 & $0,27 \mathrm{~B}$ \\
\hline \multirow[t]{2}{*}{$\mathrm{A} 2$} & $0,29 \mathrm{~B}$ & A1 & $0,20 \mathrm{~B}$ & $\mathrm{~S} 1$ & $0,34 \mathrm{~B}$ & $\mathrm{~S} 2$ & $0,19 \mathrm{C}$ \\
\hline & & & & S4 & $0,24 \mathrm{C}$ & $\mathrm{S} 1$ & $0,18 \mathrm{C}$ \\
\hline $\mathrm{CV}(\%)$ & 17,99 & & 28,22 & & 21,46 & & 24,29 \\
\hline
\end{tabular}

${ }^{1}$ Médias na mesma coluna, seguidas de mesma letra, não diferem entre si, pelo teste de Tukey $(\mathrm{P}<0,05)$.

Tabela 12 - Desdobramentos dos sistemas (S) dentro dos ambientes (A) e desdobramentos dos ambientes (A) dentro dos sistemas (S), para a área foliar (AF) em $\mathrm{mm}^{2}$ da cultivar Tudla, no período de novembro a março (NDJFM) ${ }^{1}$.

\begin{tabular}{|c|c|c|c|c|c|c|c|c|c|c|c|c|c|}
\hline \multicolumn{6}{|c|}{ Sistemas (S) dentro dos Ambientes (A) } & \multicolumn{8}{|c|}{ Ambientes (A) dentro dos Sistemas (S) } \\
\hline \multicolumn{14}{|c|}{ NDJFM - AF } \\
\hline & A1 & & A2 & & A3 & & $\mathrm{S} 1$ & & $\mathrm{~S} 2$ & & S3 & & S4 \\
\hline S4 & $41,14 \mathrm{~A}$ & S3 & $50,67 \mathrm{~A}$ & S4 & $76,48 \mathrm{~A}$ & A3 & $38,26 \mathrm{~A}$ & A1 & $32,17 \mathrm{~A}$ & A3 & $63,96 \mathrm{~A}$ & A3 & $76,48 \mathrm{~A}$ \\
\hline S3 & $41,04 \mathrm{~A}$ & S4 & $46,69 \mathrm{~A}$ & S3 & $63,96 \mathrm{~B}$ & A1 & $27,68 \mathrm{AB}$ & A3 & $28,52 \mathrm{~A}$ & $\mathrm{~A} 2$ & $50,67 \mathrm{~B}$ & $\mathrm{~A} 2$ & $46,69 \mathrm{~B}$ \\
\hline $\mathrm{S} 2$ & $32,17 \mathrm{AB}$ & $\mathrm{S} 2$ & $27,69 \mathrm{~B}$ & $\mathrm{~S} 1$ & $38,26 \mathrm{C}$ & $\mathrm{A} 2$ & $26,72 \mathrm{~B}$ & $\mathrm{~A} 2$ & $27,69 \mathrm{~A}$ & A1 & $41,04 \mathrm{~B}$ & A1 & $41,14 \mathrm{~B}$ \\
\hline $\mathrm{S} 1$ & $27,68 \mathrm{~B}$ & $\mathrm{~S} 1$ & $26,72 \mathrm{~B}$ & $\mathrm{~S} 2$ & $28,52 \mathrm{C}$ & & & & & & & & \\
\hline \multicolumn{14}{|c|}{ NDJFM - MFF } \\
\hline \multicolumn{2}{|r|}{ A1 } & \multicolumn{2}{|r|}{ A2 } & \multicolumn{2}{|r|}{ A3 } & \multicolumn{2}{|r|}{$\mathrm{S} 1$} & \multicolumn{2}{|r|}{ S2 } & \multicolumn{2}{|r|}{ S3 } & \multicolumn{2}{|r|}{ S4 } \\
\hline S3 & $0,91 \mathrm{~A}$ & S3 & $1,14 \mathrm{~A}$ & $\mathrm{~S} 4$ & $1,65 \mathrm{~A}$ & A3 & $0,76 \mathrm{~A}$ & $\mathrm{~A} 1$ & $0,71 \mathrm{~A}$ & A3 & $1,61 \mathrm{~A}$ & A3 & $1,65 \mathrm{~A}$ \\
\hline S4 & $0,87 \mathrm{~A}$ & $\mathrm{~S} 4$ & $0,96 \mathrm{~A}$ & S3 & $1,61 \mathrm{~A}$ & $\mathrm{~A} 2$ & $0,58 \mathrm{~A}$ & $\mathrm{~A} 2$ & $0,65 \mathrm{~A}$ & A2 & $1,14 \mathrm{~B}$ & $\mathrm{~A} 2$ & $0,96 \mathrm{~B}$ \\
\hline $\mathrm{S} 2$ & $0,71 \mathrm{AB}$ & $\mathrm{S} 2$ & $0,65 \mathrm{~B}$ & $\mathrm{~S} 1$ & $0,76 \mathrm{~B}$ & A1 & $0,56 \mathrm{~A}$ & A3 & $0,60 \mathrm{~A}$ & A1 & $0,91 \mathrm{~B}$ & A1 & $0,87 \mathrm{~B}$ \\
\hline $\mathrm{S} 1$ & $0,56 \mathrm{~B}$ & $\mathrm{~S} 1$ & $0,58 \mathrm{~B}$ & $\mathrm{~S} 2$ & $0,60 \mathrm{~B}$ & & & & & & & & \\
\hline \multicolumn{14}{|c|}{ NDJFM - MSF } \\
\hline \multicolumn{2}{|r|}{ A1 } & \multicolumn{2}{|r|}{$\mathrm{A} 2$} & \multicolumn{2}{|r|}{ A3 } & \multicolumn{2}{|r|}{$\mathrm{S} 1$} & \multicolumn{2}{|r|}{ S2 } & \multicolumn{2}{|r|}{ S3 } & \multicolumn{2}{|r|}{ S4 } \\
\hline S3 & $0,26 \mathrm{~A}$ & $\mathrm{~S} 3$ & $0,30 \mathrm{~A}$ & S3 & $0,41 \mathrm{~A}$ & A3 & $0,23 \mathrm{~A}$ & $\mathrm{~A} 1$ & $0,20 \mathrm{~A}$ & A3 & $0,41 \mathrm{~A}$ & A3 & $0,37 \mathrm{~A}$ \\
\hline $\mathrm{S} 2$ & $0,20 \mathrm{AB}$ & $\mathrm{S} 4$ & $0,26 \mathrm{~A}$ & $\mathrm{~S} 4$ & $0,37 \mathrm{~A}$ & $\mathrm{~A} 2$ & $0,16 \mathrm{~B}$ & $\mathrm{~A} 3$ & $0,18 \mathrm{~A}$ & A2 & $0,30 \mathrm{~B}$ & $\mathrm{~A} 2$ & $0,26 \mathrm{~B}$ \\
\hline S4 & $0,20 \mathrm{AB}$ & $\mathrm{S} 2$ & $0,18 \mathrm{~B}$ & $\mathrm{~S} 1$ & $0,23 \mathrm{~B}$ & A1 & $0,15 \mathrm{~B}$ & $\mathrm{~A} 2$ & $0,18 \mathrm{~A}$ & A1 & $0,26 \mathrm{~B}$ & A1 & $0,20 \mathrm{~B}$ \\
\hline $\mathrm{S} 1$ & $0,15 \mathrm{~B}$ & $\mathrm{~S} 1$ & $0,16 \mathrm{~B}$ & $\mathrm{~S} 2$ & $0,18 \mathrm{~B}$ & & & & & & & & \\
\hline
\end{tabular}

${ }^{1}$ Médias na mesma coluna, seguidas de mesma letra, não diferem entre si, pelo teste de Tukey $(\mathrm{P}<0,05)$. 


\section{CONCLUSÕES}

De maneira geral, houve destaque para o ambiente climatizado e o sistema de canais de $150 \mathrm{~mm}$ com vaso contendo fibra de coco, para a massa e área foliar do morangueiro.

As cultivares apresentaram maiores massas e área foliar, nos meses de maio, junho e julho, correspondendo à fase inicial de "frutos".

A cultivar Campinas, no início de produção de "frutos", apresentou melhores características foliares de área e massa fresca, nos sistemas com vaso e vertical. Na fase seguinte (pico de produção) destacou-se o sistema com vaso.

Para a cultivar Seascape, no inicio de produção de "frutos", as características foliares foram melhores nos sistemas com vaso e vertical, sendo que, no período final, esses sistemas destacaram-se apenas no ambiente climatizado. No pico de produção, o sistema com vaso apresentou os melhores resultados no ambiente climatizado para todos os parâmetros foliares e, no ambiente com aplicação de gás, somente para a área foliar.

Para a cultivar Sweet Charlie no início e final de produção os sistemas de vaso e vertical apresentam os melhores resultados, sendo que, no início, esses sistemas se destacaram nos ambiente climatizado e com aplicação de gás, e no final somente dentro do ambiente climatizado, para todas as variáveis estudadas. No pico de produção, o sistema de $150 \mathrm{~mm}$ revelou os melhores resultados dentro do ambiente climatizado, no entanto, dentro do ambiente com apenas aplicação de $\mathrm{CO}_{2}$, destacou-se somente o sistema com vaso.

Para a cultivar Tudla, no pico de produção, destacou-se o sistema com vaso e, no período final, os sistemas com vaso e vertical, nos ambiente climatizado e com aplicação de $\mathrm{CO}_{2}$.

\section{AGRADECIMENTOS}

À FAPESP (Fundação de Amparo à Pesquisa do Estado de São Paulo), pelos recursos de auxílio à pesquisa concedidos (Processo n ${ }^{\circ}$ 99/04753-4) e à CAPES (Coordenação de Aperfeiçoamento de Pessoal de Nível Superior), pela bolsa de Doutorado, tornando possível a realização deste trabalho científico.

\section{REFERÊNCIAS BIBLIOGRÁFICAS}

BENINCASA, M. M. P. Análise de crescimento de plantas. Jaboticabal: FUNEP, 1988. 42 p.

BURIOL, G. A. et al. Efeito da ventilação sobre a temperatura e umidade do ar em túneis baixos de polietileno transparente e o crescimento da alface. Revista Brasileira de Agrometeorologia, Santa Maria, v. 5, n. 1, p. 17-24, 1997.

BUTLER, L. M.; FERNANDEZ, G. E.; LOUWS, F. J. Strawberry plant growth parameters and yield among transplants of different types and from different geographic sources, grown in a plasticulture system. Hortechnology, v. 12, n. 1, p. 100-103, Jan./Mar. 2002.

CARRIJO, O. A. et al. Fibra da casca do coco verde como substrato agrícola. Horticultura Brasileira, Brasília, v. 20, n. 4, p. 533-535, dez. 2002.

CARRIJO, O. A. et al . Tomato crop production under different substrates and greenhouse models. Horticultura Brasileira, Brasília, v. 22, n. 1, 2004.

COSTA, E. Avaliação da produção do morangueiro em sistemas hidropônicos, utilizando casas de vegetação com diferentes níveis tecnológicos. $130 \mathrm{f}$. Tese (Doutorado em Construções Rurais e Ambiência) - Universidade Estadual de Campinas, Campinas, 2004.

COSTA, E.; LEAL, P. M.; CARMO JUNIOR, R. R. do. Modelo de simulação da temperatura e umidade relativa do ar no interior da estufa plástica. Revista Engenharia Agrícola, Jaboticabal, v. 24, n. 1, p. 57-67, jan./abr. 2004.

CUNHA, A. R.; ESCOBEDO, J. F. Alterações micrometeorológicas causadas pela estufa plástica e seus efeitos no crescimento e produção da cultura de pimentão. Revista Brasileira de Agrometeorologia, Santa Maria, v. 11, n. 1, p. 15-27, 2003.

FERNANDES JÚNIOR, F. Produção do morangueiro em solo, hidroponia NFT e colunas verticais com substrato. 2001. 88 f. Dissertação (Mestrado) - Instituto Agronômico de Campinas, Campinas, 2001.

GUSMÃO, M. T. A. Análise do comportamento da cultura do morangueiro (Fragaria $x$ ananassa Duch.) em condições de cultivo hidropônico. 2000. 60 f. Dissertação (Mestrado em Agronomia/Produção Vegetal) - Universidade Estadual de São Paulo, Jaboticabal, 2000.

PIRES, R. C. M.; FOLEGATTI, M. V.; PASSOS, F. A. Estimativa da área foliar de morangueiro. Horticultura Brasileira, Brasília, v. 17, n. 2, p. 86-90, 1999.

SEGOVIA, J. F. O. et al. Comparação do crescimento e desenvolvimento da alface (Lactuca sativa L.) no interior e no exterior de estufas de polietileno em Santa Maria, RS. Ciência Rural, Santa Maria, v. 27, n. 1, p. 37-41, 1997.

UNIVERSIDADE ESTADUAL PAULISTA “JÚLIO DEMESQUITA FILHO”. Departamento de Ciências Exatas. ESTAT. Versão 2.0. Jaboticabal, 1994. 